\title{
APPROXIMATION BY NORMAL DERIVATIVES OF FUNDAMENTAL SOLUTIONS OF ELLIPTIC DIFFERENTIAL OPERATOR SYSTEMS
}

\section{Ute Clauss and Uwe Hamann}

\begin{abstract}
We deal with the simultaneous approximation of a set of functions which are given on a smooth $d$-dimensional surface $\Gamma$ in $\mathbf{R}^{n}$ such that $\mathbf{R}^{n} \backslash \bar{\Gamma}$ is connected. We approximate these functions by normal derivatives of finite linear combinations of vector functions $D^{\alpha} \underline{E}_{j}\left(x-y_{k}\right)$. Here $\underline{E}_{j}$ are the columns of a fundamental solution $\underline{E}$ of a Douglis-Nirenberg-elliptic differential operator, and the sequence of points $\left(y_{k}\right)_{k=1}^{\infty} \subset \mathbf{R}^{n} \backslash \bar{\Gamma}$ is dense in $K \subset \mathbf{R}^{n} \backslash \bar{\Gamma}$ where $K$ is a $C^{1}$-smooth $(n-1)$-dimensional compact surface.
\end{abstract}

\section{Introduction}

Let $\underline{L}(D)=\left(L_{i j}(D)\right)_{i, j=1, \ldots, N}$ be a system of differential operators in $\mathbf{R}^{n}$ with constant coefficients, which is elliptic in the sense of Douglis-Nirenberg with $T=\left(t_{1}, \ldots, t_{N}\right)$ and $S=\left(s_{1}, \ldots, s_{N}\right)\left(t \stackrel{\text { def }}{=} t_{1} \geq \cdots \geq t_{N} \geq 0,0=s_{1} \geq \cdots \geq s_{N} \stackrel{\text { def }}{=} s\right)$. Hence, ord $L_{i j} \leq s_{i}+t_{j}$, and $L_{i j}=0$ if $s_{i}+t_{j}<0$.

Let $\underline{E}=\left(E_{i j}\right)_{i, j=1, \ldots, N}$ denote a fundamental solution of $\underline{L}$, and let $\underline{E}_{j}$ denote a column of $\underline{E}$.

Furthermore, let $\Gamma$ be a $C^{\infty}$-smooth $(n-1)$-dimensional bounded, and in the sense of local coordinates, open surface in $\mathbf{R}^{n}$ such that $\mathbf{R}^{n} \backslash \bar{\Gamma}$ is connected. Let $D_{\underline{n}}$ denote the derivative with respect to the direction of the normal vector at $\Gamma$.

For a given sequence of points $\left(y_{k}\right)_{k=1}^{\infty} \subset \mathbf{R}^{n} \backslash \bar{\Gamma}$, we consider finite linear combinations of vector functions of the form

$$
D^{\alpha} \underline{E}_{j}\left(x-y_{k}\right) \quad \text { where } \quad 1 \leq k<\infty ; \quad j=1, \ldots, N ; \quad 0 \leq|\alpha|<\infty .
$$

Let

$$
\underline{u}_{l, r}(x)=\sum_{k=1}^{l} \sum_{j=1}^{N} \sum_{|\alpha| \leq r} c_{k, j, \alpha} D^{\alpha} \underline{E}_{j}\left(x-y_{k}\right)
$$

be such a linear combination. We want to approximate a given system of functions on $\Gamma$ by normal derivatives of the components of $\underline{u}_{l, r}$ at the same time. Let $f_{h}^{i}$ $\left(i=1, \ldots, N^{*} ; h=0, \ldots, t_{i}+s-1\right)$ be the functions which are to be approximated. Here $N^{*}$ is the maximal integer $i$ such that $t_{i}+s-1 \geq 0$.

We will see that such approximations are possible under certain conditions. These conditions essentially refer to the location of the points $y_{k}$. The following assertion is a special case of the approximation theorem which we will prove:

$$
\begin{aligned}
& \text { Assume that the sequence }\left(y_{k}\right)_{k=1}^{\infty} \subset \mathbf{R}^{n} \backslash \bar{\Gamma} \text { is dense on a smooth } \\
& (n-1) \text {-dimensional surface } K \subset \mathbf{R}^{n} \backslash \bar{\Gamma} \text {. Then, for all systems of functions }
\end{aligned}
$$

Received March 29, 1995, revised February 6, 1996.

1991 Mathematics Subject Classification: 35A08, 35J45, 35E05.

Key words and phrases: systems of partial differential operators, ellipticity, fundamental solution, approximation. 
$\left(f_{h}^{i}\right)_{i=1, \ldots, N^{*}, h=0, \ldots, t_{i}+s-1}, f_{h}^{i} \in C(\bar{\Gamma})$, and for all $\varepsilon>0$, there exist a natural number $l$ and coefficients $c_{k, j, \alpha}\left(1 \leq k \leq l ; j=1, \ldots, N ;|\alpha| \leq t+s_{j}-1\right)$ such that

$$
\sum_{i=1}^{N^{*}} \sum_{h=0}^{t_{i}+s-1}\left\|f_{h}^{i}-\left.D_{\underline{n}}^{h} u_{l}^{i}\right|_{\Gamma}\right\|_{C(\bar{\Gamma})}<\varepsilon
$$

where

$$
\underline{u}_{l}(x)=\sum_{k=1}^{l} \sum_{j=1}^{N} \sum_{|\alpha| \leq t+s_{j}-1} c_{k, j, \alpha} \cdot D^{\alpha} \underline{E}_{j}\left(x-y_{k}\right) .
$$

Note that the surface $K$ may be arbitrarily small.

This approximation result has been formulated for simplicity for an $(n-1)$-dimensional surface $\Gamma$, but it is true in general for $d$-dimensional surfaces $(1 \leq d \leq n-1)$. Furthermore, the functions to be approximated may be chosen from different function spaces on $\Gamma$ instead of from $C(\bar{\Gamma})$.

The results of this paper can be viewed as a generalization of a theorem by Beckert [1], proved in 1960, to general elliptic systems of differential operators. Beckert dealt with approximation of functions, given on a surface $\Gamma$, by solutions of boundary-value problems, instead of by fundamental solutions. But this is strongly connected with approximation problems we deal with in this paper. Beckert considered boundary-value problems for scalar differential operators of second order. The Beckert result has been developed further by Beyer [2], Göpfert [8], Hamann [9, 11], Wildenhain [18], and Roitberg and Sheftel [16]; the latter generalized it for systems. In the papers of Browder [4,5], functions, given on a surface $\Gamma$, are approximated by normal derivatives of functions which are solutions of a scalar homogeneous elliptic equation in a fixed neighborhood of $\bar{\Gamma}$.

In papers written by Schulze and Wildenhain [17], Kupradze [14], Beyer [3], Freeden and Reuter [7], Roitberg and Roitberg [15], and Hamann [12], the problem of approximation by linear combinations of fundamental solutions, most of all for scalar differential operators or special systems of differential operators, has been investigated. A part of these results has been transfered to elliptic systems, namely for the case that $\mathbf{R}^{n} \backslash \bar{\Gamma}$ is not connected, i.e., that $\mathbf{R}^{n}$ is divided into two parts by $\Gamma$. This was done by Hamann [13] for Petrovskij-elliptic systems. Note that the cases for $\mathbf{R}^{n} \backslash \bar{\Gamma}$ connected and $\mathbf{R}^{n} \backslash \bar{\Gamma}$ not connected are fundamentally different.

An essential reason for investigating approximation by fundamental solutions in the case of a. nonconnected set $\mathbf{R}^{n} \backslash \bar{\Gamma}$ is the applicability of such approximation assertions to the construction of numerical solutions of boundary-value problems with respect to a region $\Omega$ which is bounded by $\Gamma$. In [7] and [14], such numerical constructions were carried out for the Lamé equations.

In the case of a connected set $\mathbf{R}^{n} \backslash \bar{\Gamma}$, Göpfert [8] described how the approximation assertions can be interpreted in the linear theory of elasticity (Lamé equations). He showed that displacement and stress vectors given on a surface $\Gamma$ can be approximated by influences on another surface $K$.

In the present paper, we consider the case of a connected set $\mathbf{R}^{n} \backslash \bar{\Gamma}$ for the more general class of Douglis-Nirenberg-elliptic systems. Here the results obtained by Clauss [6] are being improved.

This paper is structured in the following way. In Section 2, we introduce notations and explain assumptions for the approximation result which is given in Section 3. In 
Section 4, we state necessary lemmata and, finally, we prove the approximation result in Section 5.

\section{Definitions and notations}

2.1. Let

$$
\underline{L}(D)=\left(L_{i j}(D)\right)_{i, j=1, \ldots, N}, \quad L_{i j}(D)=\sum_{|\alpha| \leq t_{i j}} a_{\alpha}^{i j} \cdot D^{\alpha}
$$

where $\alpha=\left(\alpha_{1}, \ldots, \alpha_{n}\right), D_{k}=\frac{\partial}{\partial x_{k}}, D^{\alpha}=D_{1}^{\alpha_{1}} \ldots D_{n}^{\alpha_{n}}$, be a matrix of partial differential operators on $\mathbf{R}^{n}$ with constant coefficients. The operator $\underline{L}$ is called elliptic in the sense of Douglis-Nirenberg if there exist integers $s_{1}, \ldots, s_{N}$ and $t_{1}, \ldots, t_{N}$ such that

$$
L_{i j}(D) \equiv 0 \quad \text { if } \quad s_{i}+t_{j}<0 \quad \text { and } t_{i j} \leq s_{i}+t_{j} \quad \text { if } \quad s_{i}+t_{j} \geq 0
$$

and such that we have for the characteristic polynomial

$$
l(\underline{\xi})=\operatorname{det}\left(L_{i j}^{0}(\underline{\xi})\right)_{i, j=1, \ldots, N} \neq 0
$$

for real $\underline{\xi}=\left(\xi_{1}, \ldots, \xi_{n}\right) \neq 0$. Here

$$
L_{i j}^{0}(\underline{\xi})= \begin{cases}\sum_{|\alpha|=s_{i}+t_{j}} a_{\alpha}^{i j} \cdot \underline{\xi}^{\alpha} & \text { if } s_{i}+t_{j} \geq 0 \\ 0 & \text { if } s_{i}+t_{j}<0\end{cases}
$$

and $\underline{\xi}^{\alpha}=\xi_{1}^{\alpha_{1}} \cdots \xi_{n}^{\alpha_{n}}$.

If $\underline{L}$ is elliptic in the sense of Douglis-Nirenberg, then since $s_{i}+t_{j}=\left(s_{i}-k\right)+\left(t_{j}+k\right)$ for all integers $k$, we may assume that $\max \left\{s_{i} \mid 1 \leq i \leq N\right\}=0$. Then $\min \left\{t_{j} \mid 1 \leq\right.$ $j \leq N\} \geq 0$ and $t_{j}$ is the maximal order of differentiation of the function $u^{j}$ in $\underline{L}(D) \underline{u}$ where $\underline{u}=\left(u^{1}, \ldots, u^{N}\right)^{\top}$. By renumerating the functions $u^{j}$ and the columns of $\underline{L}(D)$, we always can get $t \stackrel{\text { def }}{=} t_{1} \geq \cdots \geq t_{N} \geq 0$ and $0=s_{1} \geq \cdots \geq s_{N} \stackrel{\text { def }}{=} s$.

\subsection{Now}

$$
\underline{L}^{*}(D)=\left(L_{j i}^{*}\right)_{i, j=1, \ldots, N}=\left(L_{i j}^{*}\right)_{i, j=1, \ldots, N}^{\top}, \quad L_{i j}^{*}(D)=\sum_{|\alpha| \leq t_{i j}}(-1)^{|\alpha|} a_{\alpha}^{i j} D^{\alpha}
$$

is the formal dual operator to $\underline{L}(D)$ in the sense of distributions. $\underline{L}^{*}(D)$ is defined by

$$
\left\langle(\underline{L u})^{\top}, \underline{v}\right\rangle \stackrel{\text { def }}{=} \sum_{i=1}^{N} \sum_{j=1}^{N} \int_{\mathbf{R}^{n}}\left(L_{i j} u^{j}\right) \cdot v^{i} d x=\sum_{j=1}^{N} \sum_{i=1}^{N} \int_{\mathbf{R}^{n}} u^{j} \cdot\left(L_{i j}^{*} v^{i}\right) d x=\left\langle\underline{u}^{\top}, \underline{L}^{*} \underline{v}\right\rangle
$$

for all $\underline{u}, \underline{v} \in\left(C_{0}^{\infty}\left(\mathbf{R}^{n}\right)\right)^{N}$.

If $\underline{L}$ is elliptic in the sense of Douglis-Nirenberg, then so is $\underline{L}^{*}$, namely with the integers $s_{i}^{*}=t_{i}-t$ and $t_{j}^{*}=s_{j}+t$ since $t_{i j}^{*}=t_{j i} \leq s_{j}+t_{i}=s_{i}^{*}+t_{j}^{*}$. We again have $t_{1}^{*} \geq \cdots \geq t_{N}^{*} \geq 0$ and $0=s_{1}^{*} \geq \cdots \geq s_{N}^{*}$.

2.3. The matrix $\underline{E}=\left(E_{i j}\right)_{i, j=1, \ldots, N}$ is called a fundamental solution of $\underline{L}$ if

$$
\underline{L E}=\left(\sum_{i=1}^{N} L_{k i} E_{i j}\right)_{k, j=1, \ldots, N}=\left(\begin{array}{ccccc}
\delta & 0 & \cdots & 0 & 0 \\
0 & \delta & & & 0 \\
\vdots & & \ddots & & \vdots \\
0 & & & \delta & 0 \\
0 & 0 & \cdots & 0 & \delta
\end{array}\right)
$$


where $\delta$ is the Dirac $\delta$-distribution. By $\underline{E}_{j}=\left(E_{1 j}, \ldots, E_{N j}\right)^{\top}$ for $j=1, \ldots, N$, we denote the columns of the fundamental solution $\underline{E}=\left(\underline{E}_{1}, \ldots, \underline{E}_{N}\right)$. The matrix function $\underline{E}^{*}$ which is defined by $\underline{E}^{*}(x)=(\underline{E}(-x))^{\top}$ is a fundamental solution of $\underline{L}^{*}$.

2.4. Let $\Gamma_{1}$ be a $C^{\infty}$-smooth $d$-dimensional $(1 \leq d \leq n-1)$, and in the sense of local coordinates on $\Gamma_{1}$, open surface. For the $C^{\infty}$-smooth $d$-dimensional open surface $\Gamma$, we assume that $\bar{\Gamma} \subset \Gamma_{1}$. Also assume that $\Gamma$ has a $C^{\infty}$-smooth $(d-1)$-dimensional boundary $\partial \Gamma$.

For $z \in \Gamma_{1}$, let $\underline{v}_{1}(z), \ldots, \underline{v}_{d}(z)$ be $d$ linear independent unit vectors which span the tangent plane at $z$, and let $\underline{v}_{d+1}(z), \ldots, \underline{v}_{n}(z)$ be $(n-d)$ linear independent unit vectors which span the normal plane at $z$. Assume that the components $v_{i k}$ of $\underline{v}_{i}$ $(i, k=1, \ldots, n)$ are in $C^{\infty}\left(\Gamma_{1}\right)$.

For a fixed point $z \in \Gamma_{1}$, let

$$
\left(D_{\underline{v}_{i}(z)} f\right)(x)=\left(\frac{\partial}{\partial \underline{v}_{i}(z)} f\right)(x)=\sum_{k=1}^{n} v_{i k}(z) \cdot\left(\frac{\partial}{\partial x_{k}} f\right)(x)
$$

be the directional derivative of $f$ at the point $x \in \mathbf{R}^{n}$ with respect to the direction of $\underline{v}_{i}(z)$.

In the same way, let

$$
\left(D_{\underline{v}(z)}^{\alpha} f\right)(x)=\frac{\left(\partial^{|\alpha|} f\right)(x)}{\partial \underline{v}_{1}^{\alpha_{1}}(z) \cdots \partial \underline{v}_{n}^{\alpha_{n}}(z)}
$$

for $\alpha=\left(\alpha_{1}, \ldots, \alpha_{n}\right)$. Note that $D_{\underline{v}(z)}^{\alpha}$ is applied with respect to the variable $x$.

Furthermore, let

$$
\left.D_{\underline{v}}^{\alpha} f\right|_{\Gamma}=\left\{\left(D_{\underline{v}(z)}^{\alpha} f\right)(x) \mid x=z \in \Gamma\right\} \text {. }
$$

For $\alpha=\alpha^{\prime \prime}=\left(0, \ldots, 0, \alpha_{d+1}, \ldots, \alpha_{n}\right),\left.D_{\underline{v}}^{\alpha^{\prime \prime}} f\right|_{\Gamma}$ contains only derivatives in normal directions of $\Gamma$. Hence, we may call $\left.D_{\underline{v}}^{\alpha^{\prime \prime}} f\right|_{\Gamma}$ the normal derivative of order $\alpha^{\prime \prime}$ of $f$ on $\Gamma$.

2.5. The spaces from which we may choose the functions on $\Gamma$ we want to approximate are Banach spaces with certain properties. We now formulate two conditions on a Banach space $D(\Gamma)$.

(a1) There is an integer $r_{1}$ such that $C^{r_{1}}(\bar{\Gamma})$ is continuously embedded in $D(\Gamma)$.

(a2) $\overline{C^{\infty}(\bar{\Gamma})}=D(\Gamma)$, the closure being taken in $D(\Gamma)$.

These conditions are not very strong. There are a variety of spaces which satisfy these conditions. Some examples are

- the space of $k$-times continously differentiable functions $C^{k}(\bar{\Gamma})$ for all integers $k \geq 0$,

- the Sobolev-Slobodeckij-space $W^{p, s}(\Gamma)$ for $0 \leq s<\infty, 1 \leq p<\infty, s, p \in \mathbf{R}$,

- the dual space $\left(W^{p, s}(\Gamma)\right)^{\prime}$ of $W^{p, s}(\Gamma)$ for $0 \leq s<\infty, 1<p<\infty, s, p \in \mathbf{R}$.

The Hölder spaces $C^{k, \lambda}(\bar{\Gamma})(k \in \mathbf{N} ; 0<\lambda \leq 1)$ do not satisfy condition (a2).

\section{Approximation result}

First, we want to summarize the suppositions.

1. Let $\Gamma$ be a $C^{\infty}$-smooth $d$-dimensional surface such that $\mathbf{R}^{n} \backslash \bar{\Gamma}$ is connected. By $\alpha^{\prime \prime}$, we always denote multi-indices of the following form: $\alpha^{\prime \prime}=\left(0, \ldots, 0, \alpha_{d+1}, \ldots, \alpha_{n}\right)$. $\left.D_{\underline{v}}^{\alpha^{\prime \prime}} f\right|_{\Gamma}$ is the normal derivative of order $\alpha^{\prime \prime}$ of the function $f$ on $\Gamma$. 
2. Let $\underline{L}(D)=\left(L_{i j}(D)\right)_{i, j=1, \ldots, N}$ be a system of differential operators on $\mathbf{R}^{n}$ with constant coefficients which is elliptic in the sense of Douglis-Nirenberg with $T=\left(t_{1}, \ldots, t_{N}\right)$ and $S=\left(s_{1}, \ldots, s_{N}\right)$. Here $t=t_{1} \geq \cdots \geq t_{N} \geq 0$ and $0=s_{1} \geq \cdots \geq$ $s_{N}=s$. We assume $t+s-1 \geq 0$.

3. Let $\underline{E}=\left(E_{i j}\right)_{i, j=1, \ldots, N}$ denote a fundamental solution of $\underline{L}$, and let $\underline{E}_{j}$ denote the columns of $\underline{E}$.

4. We denote the maximal integer $i$ with $t_{i}+s-1 \geq 0$ by $N^{*}$. Hence, we have $t_{i}+s-1 \geq 0$ for all $i=1, \ldots, N^{*}$. Let $D_{i, \beta^{\prime \prime}}(\Gamma)\left(i=1, \ldots, N^{*} ;\left|\beta^{\prime \prime}\right| \leq t_{i}+s-1\right.$, $\left.\beta^{\prime \prime}=\left(0, \ldots, 0, \beta_{d+1}, \ldots, \beta_{n}\right)\right)$ be Banach spaces which satisfy the conditions (a1) and (a2).

Theorem. Let $K \subset \mathbf{R}^{n} \backslash \bar{\Gamma}$ be a $C^{1}$-smooth $(n-1)$-dimensional compact surface. Let the sequence of points $\left(y_{k}\right)_{k=1}^{\infty} \subset \mathbf{R}^{n} \backslash \bar{\Gamma}$ be dense in $K$. Then, for all vector functions

$$
\underline{f}=\left(\underline{f}^{1}, \ldots, \underline{f}^{N^{*}}\right)^{\top}, \quad \underline{f}^{i}=\left(f_{\beta^{\prime \prime}}^{i}\right)_{\left|\beta^{\prime \prime}\right| \leq t_{i}+s-1}, \quad f_{\beta^{\prime \prime}}^{i} \in D_{i, \beta^{\prime \prime}}(\Gamma),
$$

with $\beta^{\prime \prime}=\left(0, \ldots, 0, \beta_{d+1}, \ldots, \beta_{n}\right)$, there are numbers $c_{k, j, \alpha}^{(l)}(1 \leq l<\infty, 1 \leq k \leq l$, $\left.j=1, \ldots, N,|\alpha| \leq t+s_{j}-1\right)$, such that

$$
\lim _{l \rightarrow \infty} \sum_{i=1}^{N^{*}} \sum_{\left|\beta^{\prime \prime}\right| \leq t_{i}+s-1}\left\|\left.D_{\underline{v}}^{\beta^{\prime \prime}} u_{l}^{i}\right|_{\Gamma}-f_{\beta^{\prime \prime}}^{i}\right\|_{D_{i, \beta^{\prime \prime}}(\Gamma)}=0
$$

for

$$
\left(\begin{array}{c}
u_{l}^{1}(x) \\
\vdots \\
u_{l}^{N}(x)
\end{array}\right)=\underline{u}_{l}(x)=\sum_{k=1}^{l} \sum_{j=1}^{N} \sum_{|\alpha| \leq t+s_{j}-1} c_{k, j, \alpha}^{(l)} \cdot D^{\alpha} \underline{E}_{j}\left(x-y_{k}\right) .
$$

Remarks. 1. The assumption of the $C^{1}$-smoothness of $K$ can be weakened (see Lemma 3 and [12], Theorem 1). Only the $(n-1)$-dimensionality is the essential property of $K$.

2. The points $y_{k}$ may, but don't have to, lie on $K$.

3. The compact set $K$ may be arbitrarily small.

4. Similar to the Theorems 2 to 6 in [13], one may consider different constellations of the points $\left(y_{k}\right)_{k=1}^{\infty}$. Then, one obtains analogous statements regarding the summation over $\alpha$ in $\underline{u}_{l}$.

5. It is not hard to show that normal derivatives can be replaced by nontangential derivatives.

\section{Preparation for the proof}

4.1. If $X$ is a normed space, we denote by $X^{\prime}$ its dual space and by $\langle f, F\rangle$ the pairing between $f \in X$ and $F \in X^{\prime}$.

For normed spaces $X$ and $Y$, let $L(X, Y)$ be the set of all continuous linear operators mapping $X$ into $Y$. For $B \in L(X, Y)$, let $B^{\prime} \in L\left(Y^{\prime}, X^{\prime}\right)$ denote the dual operator which is defined by the equation

$$
\langle B f, F\rangle=\left\langle f, B^{\prime} F\right\rangle \text { for all } f \in X, F \in X^{\prime} .
$$

Furthermore, by $\langle f, F\rangle$ we also denote the pairing between an element $f$ of $C_{0}^{\infty}\left(\mathbf{R}^{n}\right)$ and a distribution $F \in D^{\prime}\left(\mathbf{R}^{n}\right)$. 
Let $X_{1}, \ldots, X_{N}$ be normed spaces and $X_{1}^{\prime}, \ldots, X_{N}^{\prime}$ their dual spaces. For $\underline{g}=$ $\left(g_{1}, \ldots, g_{N}\right)^{\top}, g_{j} \in X_{j}^{\prime}$, and $\underline{f}=\left(f_{1}, \ldots, f_{N}\right)^{\top}, f_{j} \in X_{j}$, we define

$$
\left\langle\underline{f}^{\top}, \underline{g}\right\rangle=\sum_{j=1}^{N}\left\langle f_{j}, g_{j}\right\rangle .
$$

For a matrix $\underline{A}=\left(a_{i j}\right)_{i=1, \ldots, M, j=1, \ldots, N}$ with $a_{i j} \in X_{j}(i=1, \ldots, M ; j=1, \ldots, N)$, let

$$
\langle\underline{A}, \underline{g}\rangle=\sum_{j=1}^{N}\left(\begin{array}{c}
\left\langle a_{1 j}, g_{j}\right\rangle \\
\left\langle a_{2 j}, g_{j}\right\rangle \\
\vdots \\
\left\langle a_{M j}, g_{j}\right\rangle
\end{array}\right) .
$$

If the $a_{i j}$ 's are distributions in $D^{\prime}\left(\mathbf{R}^{n}\right)$, and the $g_{j}$ 's are distributions with compact support, we define the convolution of $\underline{A}$ with $\underline{g}$ by

$$
\underline{A} * \underline{g} \stackrel{\text { def }}{=} \sum_{j=1}^{N}\left(\begin{array}{c}
a_{1 j} * g_{j} \\
a_{2 j} * g_{j} \\
\vdots \\
a_{M j} * g_{j}
\end{array}\right) .
$$

If $\underline{A}=\underline{E}$ is a fundamental solution of $\underline{L}$, then

$$
\underline{L}(\underline{E} * \underline{g})=\underline{g} .
$$

4.2. Let $A^{t}$ be function spaces where $t$ is a parameter which specifies the space $A^{t}$, for instance, $C^{t}(\bar{\Omega})$ or $W^{p, t}\left(\mathbf{R}^{n}\right)$. If $V=\left(v_{1}, \ldots, v_{N}\right)$ is an $N$-tuple of integers or of real numbers and if $k$ is a integer or a real number, we define $V+k=\left(v_{1}+k, \ldots, v_{N}+k\right)$ and

$$
A^{V+k}=A^{v_{1}+k} \times \cdots \times A^{v_{N}+k} .
$$

For instance, we have $W^{p, k_{0}+T}\left(\mathbf{R}^{n}\right)=W^{p, k_{0}+t_{1}}\left(\mathbf{R}^{n}\right) \times \cdots \times W^{p, k_{0}+t_{N}}\left(\mathbf{R}^{n}\right)$ and $C^{r_{0}-S+s}\left(\mathbf{R}^{n}\right)=C^{r_{0}-s_{1}+s}\left(\mathbf{R}^{n}\right) \times \cdots \times C^{r_{0}-s_{N}+s}\left(\mathbf{R}^{n}\right)$.

4.3. For an open set $\Omega \subset \mathbf{R}^{n}$ and $0 \leq k<\infty$, we define

$$
C^{0, k}(\Omega)=\overline{C_{0}^{\infty}(\Omega)}
$$

the closure being taken in the norm of $C^{k}(\Omega)$. The elements of $\left(C^{0, k}(\Omega)\right)^{\prime}$ are distributions of order $k$ on $\Omega$. Obviously, for $f \in C^{0, k}(\Omega)$ and $|\alpha| \leq k$, we have $\left.D^{\alpha} f\right|_{\partial \Omega}=0$. Now let $\Gamma$ be a $C^{\infty}$-smooth, $d$-dimensional surface and $\Omega=\mathbf{R}^{n} \backslash \bar{\Gamma}$.

Lemma 1. $C^{0, k}\left(\mathbf{R}^{n} \backslash \bar{\Gamma}\right)=\left\{f \in C^{0, k}\left(\mathbf{R}^{n}\right)\left|D_{\underline{v}}^{\alpha^{\prime \prime}} f\right|_{\Gamma}=0\right.$ for $\left|\alpha^{\prime \prime}\right| \leq k, \alpha^{\prime \prime}=$ $\left.\left(0, \ldots, 0, \alpha_{d+1}, \ldots, \alpha_{n}\right)\right\}$.

The proof for $(n-1)$-dimensional surfaces $\Gamma$ can be found in [11] (Lemma 5). Since the $(n-1)$-dimensionality has not been used there, this result can be proved for $d$-dimensional sufaces completely analogously (see also [10], Lemma 2.18). 
4.4. If $\Omega \subset \mathbf{R}^{n}$ is an open set, we denote by $W^{k, p}(\Omega)(k \geq 0 ; 1<p<\infty)$ the classical Sobolev spaces. Let $W_{0}^{k, p}(\Omega)$ be the closure of $C_{0}^{\infty}(\Omega)$ in $W^{k, p}(\Omega)$. The following holds: $W^{k, p}\left(\mathbf{R}^{n}\right)=W_{0}^{k, p}\left(\mathbf{R}^{n}\right)$. Furthermore, let $W^{-k, p^{\prime}}(\Omega)=\left(W_{0}^{k, p}(\Omega)\right)^{\prime}$ $\left(k \geq 0 ; p^{\prime}=\frac{p}{p-1}\right)$. For $-\infty<k<\infty$ and $1<p<\infty$, we define

$$
W_{l o c}^{k, p}\left(\mathbf{R}^{n}\right)=\left\{f \in D^{\prime}\left(\mathbf{R}^{n}\right)|f|_{\omega} \in W^{k, p}(\omega) \text { for all bounded open sets } \omega \subset \mathbf{R}^{n}\right\} .
$$

The operator $\underline{L}(D)$, for instance, maps $W^{k_{0}+T, p}\left(\mathbf{R}^{n}\right)\left(-\infty<k_{0}<\infty\right)$ into $W^{k_{0}-S, p}\left(\mathbf{R}^{n}\right)$. The next lemma follows from well-known regularity theorems.

Lemma 2. Let $\underline{L}(D)=\left(L_{i j}(D)\right)_{i, j=1, \ldots, N}$ be elliptic in the sense of Douglis-Nirenberg with $T=\left(t_{1}, \ldots, t_{N}\right)$ and $S=\left(s_{1}, \ldots, s_{N}\right)$. Further, let $\underline{E}=\left(E_{i j}\right)_{i, j=1, \ldots, N}$ be $a$ fundamental solution of $\underline{L}$. If $\underline{f} \in W^{k_{0}-S, p}\left(\mathbf{R}^{n}\right)\left(-\infty<k_{0}<\infty\right)$ has compact support, then $\underline{E} * \underline{f} \in W_{l o c}^{k_{0}+T, p}\left(\mathbf{R}^{n}\right)$.

4.5. The following lemma has been proved in [13] (Lemma 13). By $H_{n-1}(K)$, we denote the $(n-1)$-dimensional Hausdorff measure of a set $K \subset \mathbf{R}^{n}$.

Lemma 3. Let $\Omega \subset \mathbf{R}^{n}$ be a domain and $K \subset \Omega$ a compact set with the following properties:

1. There exists an open ball $B$, which is divided by $K$ into two non-empty disjoint open sets.

2. $H_{n-1}(K \cap B)<\infty$.

Furthermore, let $\underline{L}(D)=\left(L_{i j}(D)\right)_{i, j=1, \ldots, N}$ be elliptic in the sense of Douglis-Nirenberg with $T=\left(t_{1}, \ldots, t_{N}\right)$ and $S=\left(s_{1}, \ldots, s_{N}\right)$. Assume that the coefficients of $L_{i j}$ are constants. Then the Cauchy problem

$$
\underline{L u}=\underline{0} \text { in } \Omega,\left.\quad D^{\alpha} u^{j}\right|_{K}=0 \text { for }|\alpha| \leq t_{j}-1 \quad(j=1, \ldots, N)
$$

has only the solution $\underline{u} \equiv \underline{0}$ in $\Omega$.

Note that any $C^{1}$-smooth surface $K$ satisfies the two conditions of this lemma.

4.6. Let $z \in \Gamma_{1}$ be a fixed point. Then the directional derivatives $\frac{\partial}{\partial \underline{v}_{i}(z)}(i=1, \ldots, n)$ have the representation

$$
\frac{\partial}{\partial \underline{v}_{i}(z)}=\sum_{k=1}^{n} v_{i k}(z) \frac{\partial}{\partial x_{k}}
$$

Here $\underline{v}_{i}=\left(v_{i 1}, \ldots, v_{i n}\right)$. Further, we get

$$
\frac{\partial}{\partial x_{k}}=\sum_{i=1}^{n} w_{k i}(z) \frac{\partial}{\partial \underline{v}_{i}(z)} \quad(k=1, \ldots, n)
$$

where $\underline{W}(z)=\left(w_{k i}(z)\right)_{k, i=1, \ldots, n}$ is the inverse matrix of $\left(v_{i k}\right)_{i, k=1, \ldots, n}$. This representation of $\frac{\partial}{\partial x_{k}}$ is possible because the vectors $\underline{v}_{1}(z), \ldots, \underline{v}_{n}(z)$ are linearly independent. Hence, by defining

$$
D=\left(\frac{\partial}{\partial x_{1}}, \ldots, \frac{\partial}{\partial x_{n}}\right)^{\top} \text { and } D_{\underline{v}(z)}=\left(\frac{\partial}{\partial \underline{v}_{1}(z)}, \ldots, \frac{\partial}{\partial \underline{v}_{n}(z)}\right)^{\top}
$$

we get

$$
D=\underline{W}(z) \cdot D_{\underline{v}(z)} \text { and } D^{\alpha}=\left(\underline{W}(z) \cdot D_{\underline{v}(z)}\right)^{\alpha} .
$$


Thus, we can represent the differential operators $L_{i j}$ by means of directional derivatives in the direction of the vectors $\underline{v}_{1}(z), \ldots, \underline{v}_{n}(z)$ with respect to a fixed point $z \in \Gamma_{1}$ :

$$
\begin{aligned}
L_{i j}(D) & =\sum_{|\alpha| \leq t_{i j}} a_{\alpha}^{i j} \cdot D^{\alpha}=\sum_{|\alpha| \leq t_{i j}} a_{\alpha}^{i j}\left(\underline{W}(z) \cdot D_{\underline{v}(z)}\right)^{\alpha} \\
& =\sum_{|\alpha| \leq t_{i j}} \tilde{a}_{\alpha}^{i j} \cdot D_{\underline{v}(z)}^{\alpha}=\widetilde{L_{i j}}\left(z, D_{\underline{v}(z)}\right) .
\end{aligned}
$$

Let $\underline{\mu}=\left(\mu_{1}, \ldots, \mu_{n}\right)^{\top}$ be an arbitrary vector, and let $\underline{\xi}=\underline{W}(z) \underline{\mu}$. We obtain

$$
\begin{aligned}
l(\underline{\xi}) & =\operatorname{det}\left(L_{i j}^{0}(\underline{\xi})\right)_{i, j=1, \ldots, N}=\operatorname{det}\left(L_{i j}^{0}(\underline{W}(z) \underline{\mu})\right)_{i, j=1, \ldots, N} \\
& =\operatorname{det}\left(\widetilde{L_{i j}^{0}}(z, \underline{\mu})\right)_{i, j=1, \ldots, N}=\tilde{l}(z, \underline{\mu}) .
\end{aligned}
$$

If $\underline{\mu}$ is a vector such that $\tilde{l}(z, \underline{\mu})=0$, then $l(\underline{\xi})=0$. Since $\underline{L}$ is elliptic, we get $\underline{\xi}=\underline{0}$, and hence $\underline{0}=\underline{W}(z) \cdot \underline{\mu}$. Since $\underline{W}(z)$ is a non-singular matrix, $\underline{\mu}=\underline{0}$ follows. Hence, $\operatorname{det}\left(\widetilde{L_{i j}^{0}}(z, \underline{\mu})\right)_{i, j=1, \ldots, N}$ only vanishes for the zero vector.

Thus, for $\underline{\mu}^{*}=(0, \ldots, 0,1)^{\top}$,

$$
\operatorname{det}\left(\widetilde{L_{i j}^{0}}\left(z, \underline{\mu}^{*}\right)\right)_{i, j=1, \ldots, N} \neq 0
$$

Furthermore, we have

$$
\widetilde{L_{i j}^{0}}\left(z, \underline{\mu}^{*}\right)=\tilde{a}_{\left(0, \ldots, 0, s_{i}+t_{j}\right)}^{i j}(z)
$$

for $i, j$ with $s_{i}+t_{j} \geq 0$. For $s_{i}+t_{j}<0, \widetilde{L_{i j}^{0}}\left(z, \underline{\mu}^{*}\right) \equiv 0$. Then we formally set $\tilde{a}_{\left(0, \ldots, 0, s_{i}+t_{j}\right)}^{i j}(z)=0$. We obtain

$$
\tilde{l}\left(z, \underline{\mu}^{*}\right)=\operatorname{det}\left(\tilde{a}_{\left(0, \ldots, 0, s_{i}+t_{j}\right)}^{i j}(z)\right)_{i, j=1, \ldots, N} \neq 0
$$

for all $z \in \Gamma_{1}$.

Since $\bar{\Gamma} \subset \Gamma_{1}$ is compact, we get

$$
\left|\operatorname{det}\left(\tilde{a}_{\left(0, \ldots, 0, s_{i}+t_{j}\right)}^{i j}(z)\right)_{i, j=1, \ldots, N}\right| \geq c>0
$$

for all $z \in \Gamma$ for a constant $c$.

4.7. The following lemma has been proved in [10] (Lemma 2.14).

Lemma 4. Let $k \geq 0$ be an integer. Then for each tuple of functions

$$
\underline{g}=\left(g_{\alpha^{\prime \prime}}\right)_{\left|\alpha^{\prime \prime}\right| \leq k} \in \prod_{\left|\alpha^{\prime \prime}\right| \leq k} C^{k-\left|\alpha^{\prime \prime}\right|}(\bar{\Gamma})
$$

$\left(\alpha^{\prime \prime}=\left(0, \ldots, 0, \alpha_{d+1}, \ldots, \alpha_{n}\right)\right)$, there exists a function $w \in C_{0}^{k}\left(\mathbf{R}^{n}\right)$ such that

$$
\left.D_{\underline{v}}^{\alpha^{\prime \prime}} w\right|_{\Gamma}=g_{\alpha^{\prime \prime}} \text { for }\left|\alpha^{\prime \prime}\right| \leq k .
$$

From this lemma and the considerations in 4.6, we derive the next lemma.

For $i$ with $1 \leq i \leq N$, we define $N_{i}$ to be the maximal integer $j$ such that $s_{i}+t_{j} \geq 0$. Hence, we have $s_{i}+t_{j} \geq 0$ for $j=1, \ldots, N_{i}$. 
Lemma 5. Let $\beta_{(0)}^{\prime \prime}=\left(0, \ldots, 0, \beta_{d+1}^{(0)}, \ldots, \beta_{n}^{(0)}\right)$ be a fixed multi-index and let $i_{0}, 1 \leq$ $i_{0} \leq N$, be a fixed integer. Futhermore, let $i^{*}$ be the smallest integer $i$ such that $\beta_{n}^{(0)}+s_{i_{0}}-s_{i} \geq 0$. Then, for each function $g \in C^{r}(\bar{\Gamma})$ ( $r$ integer, $\left.r \geq 0\right)$, there exist functions $u^{j} \in C_{0}^{\left|\beta_{0}^{\prime \prime}\right|+s_{i_{0}}+t_{j}+r}\left(\mathbf{R}^{n}\right), j=1, \ldots, N_{i^{*}}$, such that

1.

$$
\begin{gathered}
\left.\sum_{j=1}^{N_{i}} \tilde{a}_{\left(0, \ldots, 0, s_{i}+t_{j}\right)}^{i j}(z) \cdot D_{\underline{v}(z)}^{\left(0, \ldots, 0, \beta_{d+1}^{(0)}, \ldots, \beta_{n-1}^{(0)}, \beta_{n}^{(0)}+s_{i_{0}}+t_{j}\right)} u^{j}(x)\right|_{x=z \in \Gamma} \\
=\left\{\begin{array}{ll}
g & \text { for } i=i_{0} \\
0 & \text { for } i \neq i_{0}
\end{array} \quad\left(i=i^{*}, \ldots, N\right),\right.
\end{gathered}
$$

2. $\left.D_{\underline{v}}^{\gamma^{\prime \prime}} u^{j}\right|_{\Gamma}=0$ for $j=1, \ldots, N_{i^{*}}$ and $\gamma^{\prime \prime}$ such that $\left|\gamma^{\prime \prime}\right| \leq\left|\beta_{(0)}^{\prime \prime}\right|+s_{i_{0}}+t_{j}$ and $\gamma^{\prime \prime} \neq\left(0, \ldots, 0, \beta_{d+1}^{(0)}, \ldots, \beta_{n-1}^{(0)}, \beta_{n}^{(0)}+s_{i_{0}}+t_{j}\right)$.

Proof. Since $\operatorname{det}\left(\tilde{a}_{\left(0, \ldots, 0, s_{i}+t_{j}\right)}^{i j}(z)\right)_{i, j=1, \ldots, N} \neq 0$ for all $z \in \bar{\Gamma}$ (we again set $\tilde{a}_{\left(0, \ldots, 0, s_{i}+t_{j}\right)}^{i j}(z) \stackrel{\text { def }}{=} 0$ for $\left.s_{i}+t_{j}<0\right)$, the linear system of equations

$$
\sum_{j=1}^{N_{i}} \tilde{a}_{\left(0, \ldots, 0, s_{i}+t_{j}\right)}^{i j}(z) \cdot w^{j}(z)=\left\{\begin{array}{ll}
g(z) & \text { for } i=i_{0} \\
0 & \text { for } i \neq i_{0}
\end{array}(i=1, \ldots, N)\right.
$$

has exactly one solution $\underline{w}(z)=\left(w^{1}(z), \ldots, w^{N}(z)\right)$ for each $z \in \bar{\Gamma}$. Since $g \in C^{r}(\bar{\Gamma})$, $\left(\operatorname{det}\left(\tilde{a}_{\left(0, \ldots, 0, s_{i}+t_{j}\right)}^{i j}\right)_{i, j=1, \ldots, N}\right)^{-1} \in C^{\infty}(\bar{\Gamma})$ and $\tilde{a}_{\alpha}^{i j} \in C^{\infty}(\bar{\Gamma})$, we obtain $w^{j} \in C^{r}(\bar{\Gamma})$.

Lemma 4 yields the existence of functions $u^{j} \in C_{0}^{\left|\beta_{(0)}^{\prime \prime}\right|+s_{i_{0}}+t_{j}+r}\left(\mathbf{R}^{n}\right), j=1, \ldots, N_{i^{*}}$, such that

$$
\left.D_{\underline{v}}^{\left(0, \ldots, 0, \beta_{d+1}^{(0)}, \ldots, \beta_{n-1}^{(0)}, \beta_{n}^{(0)}+s_{i_{0}}+t_{j}\right)} u^{j}\right|_{\Gamma}=w^{j} \text { and }\left.D_{\underline{v}}^{\gamma^{\prime \prime}} u^{j}\right|_{\Gamma}=0
$$

for all $\gamma^{\prime \prime}$ with $\left|\gamma^{\prime \prime}\right| \leq\left|\beta_{(0)}^{\prime \prime}\right|+s_{i_{0}}+t_{j}$ and $\gamma^{\prime \prime} \neq\left(0, \ldots, 0, \beta_{d+1}^{(0)}, \ldots, \beta_{n-1}^{(0)}, \beta_{n}^{(0)}+s_{i_{0}}+t_{j}\right)$. Now (1) and (2) yield the desired properties 1 and 2 .

4.8. The proof of the approximation theorem is based on the following lemma which follows from the Theorem of Hahn-Banach.

Lemma 6. Let $X$ be a normed space and $X_{0}$ be a linear subset of $X$. Let $x^{\prime} \in X^{\prime}$. If

$$
\left\langle x_{0}, x^{\prime}\right\rangle=0 \text { for all } x_{0} \in X_{0}
$$

implies $x^{\prime}=0$, then $\overline{X_{0}}=X$.

\section{Proof of the approximation result}

Before we begin to prove the approximation result, we define a restriction operator so that we can formulate the result a bit differently. Let $N^{*}$ be the maximal integer $i$ such that $t_{i}+s-1 \geq 0$. Let

$$
\underline{R}_{T+s-1} \underline{u}=\left(\left(\left.D_{\underline{v}}^{\beta^{\prime \prime}} u^{1}\right|_{\Gamma}\right)_{\left|\beta^{\prime \prime}\right| \leq t_{1}+s-1}, \ldots,\left(\left.D_{\underline{v}}^{\beta^{\prime \prime}} u^{N^{*}}\right|_{\Gamma}\right)_{\left|\beta^{\prime \prime}\right| \leq t_{N^{*}+s-1}}\right)^{\top}
$$

for vector functions $\underline{u}=\left(u^{1}, \ldots, u^{N}\right)^{\top}$ sufficiently smooth in a neighborhood of $\bar{\Gamma}$ $\left(u^{N^{*}+1}, \ldots, u^{N}\right.$ are not considered in $\left.\underline{R}_{T+s-1}\right)$. For abbreviation, we write $\underline{R}$ instead of $\underline{R}_{T+s-1}$. Now the claim of the approximation result reads: 
The closure of the linear hull of

$$
\left\{\underline{R}\left(D^{\alpha} \underline{E}_{j}\left(x-y_{k}\right)\right)|1 \leq j \leq N ;| \alpha \mid \leq t+s_{j}-1 ; k=1,2, \ldots\right\}
$$

is

$$
\prod_{i=1}^{N^{*}}\left(\prod_{\left|\beta^{\prime \prime}\right| \leq t_{i}+s-1} D_{i, \beta^{\prime \prime}}(\Gamma)\right)
$$

We will prove this formulation. The proof consists of 15 steps.

Step 1. Let

$$
\underline{T}=\left(\left(T_{\beta^{\prime \prime}}^{1}\right)_{\left|\beta^{\prime \prime}\right| \leq t_{1}+s-1}, \ldots,\left(T_{\beta^{\prime \prime}}^{N^{*}}\right)_{\left|\beta^{\prime \prime}\right| \leq t_{N^{*}}+s-1}\right)^{\top}
$$

be a linear continuous functional on $\prod_{i=1}^{N^{*}}\left(\prod_{\left|\beta^{\prime \prime}\right| \leq t_{i}+s-1} D_{i, \beta^{\prime \prime}}(\Gamma)\right)$ such that

$$
\left\langle\left(\underline{R}\left(\left(D^{\alpha} \underline{E}_{j}\right)\left(x-y_{k}\right)\right)\right)^{\top}, \underline{T}(x)\right\rangle=0
$$

for $j=1, \ldots, N,|\alpha| \leq t+s_{j}-1$ and $k=1,2, \ldots$. Here,

$$
\begin{aligned}
& \left\langle\left(\underline{R}\left(\left(D^{\alpha} \underline{E}_{j}\right)\left(x-y_{k}\right)\right)\right)^{\top}, \underline{T}(x)\right\rangle \\
& \quad=\sum_{i=1}^{N^{*}} \sum_{\left|\beta^{\prime \prime}\right| \leq t_{i}+s-1}\left\langle\left. D_{\underline{v}}^{\beta^{\prime \prime}}\left(D^{\alpha} E_{i j}\left(x-y_{k}\right)\right)\right|_{x \in \Gamma}, T_{\beta^{\prime \prime}}^{i}(x)\right\rangle .
\end{aligned}
$$

From (3), we will conclude that $\underline{T}=\underline{0}$. Then Lemma 6 gives the claim.

Step 2. Because of condition (a1), there exists an integer $r_{1} \geq 0$ such that

$$
C^{r_{1}}(\bar{\Gamma}) \hookrightarrow D_{i, \beta^{\prime \prime}}(\Gamma)
$$

for all $i=1, \ldots, N^{*}$ and $\beta^{\prime \prime}=\left(0, \ldots, 0, \beta_{d+1}, \ldots, \beta_{n}\right)$ such that $\left|\beta^{\prime \prime}\right| \leq t_{i}+s-1$. Also since every integer greater than $r_{1}$ fulfills this condition, we choose $r_{0}=\max \left\{r_{1},-s+\right.$ $1\}$, so that $r_{0}+s-1 \geq 0$ always. Hence,

$$
\prod_{i=1}^{N^{*}}\left(\prod_{\left|\beta^{\prime \prime}\right| \leq t_{i}+s-1}\left(D_{i, \beta^{\prime \prime}}(\Gamma)\right)^{\prime}\right) \hookrightarrow \prod_{i=1}^{N^{*}}\left(\prod_{\left|\beta^{\prime \prime}\right| \leq t_{i}+s-1}\left(C^{r_{0}}(\bar{\Gamma})\right)^{\prime}\right) .
$$

Since $\underline{T}$ is an element of $\prod_{i=1}^{N^{*}}\left(\prod_{\left|\beta^{\prime \prime}\right| \leq t_{i}+s-1}\left(D_{i, \beta^{\prime \prime}}(\Gamma)\right)^{\prime}\right)$, we now can consider $\underline{T}$ as an element of $\prod_{i=1}^{N^{*}}\left(\prod_{\left|\beta^{\prime \prime}\right| \leq t_{i}+s-1}\left(C^{r_{0}}(\bar{\Gamma})\right)^{\prime}\right)$. For the restriction operator, we have

$$
\underline{R} \in L\left(C^{0, r_{0}+T+s-1}\left(\mathbf{R}^{n}\right), \prod_{i=1}^{N^{*}}\left(\prod_{\left|\beta^{\prime \prime}\right| \leq t_{i}+s-1} C^{r_{0}}(\bar{\Gamma})\right)\right) .
$$

Hence, for the dual operator, we have

$$
\underline{R}^{\prime} \in L\left(\prod_{i=1}^{N^{*}}\left(\prod_{\left|\beta^{\prime \prime}\right| \leq t_{i}+s-1}\left(C^{r_{0}}(\bar{\Gamma})\right)^{\prime}\right),\left(C^{0, r_{0}+T+s-1}\left(\mathbf{R}^{n}\right)\right)^{\prime}\right) .
$$

Thus, $\underline{R}^{\prime} \underline{T}$ is an element of $\left(C^{0, r_{0}+T+s-1}\left(\mathbf{R}^{n}\right)\right)^{\prime}$.

Let $\underline{\phi}=\left(\phi_{1}, \ldots, \phi_{N}\right)^{\top} \in\left(C_{0}^{\infty}\left(\mathbf{R}^{n} \backslash \bar{\Gamma}\right)\right)^{N}$ an arbitrary vector function. Then

$$
\left\langle\underline{\phi}^{\top}, \underline{R}^{\prime} \underline{T}\right\rangle=\left\langle(\underline{R} \underline{\phi})^{\top}, \underline{T}\right\rangle=0 ;
$$

hence, supp $\underline{R}^{\prime} \underline{T} \subset \bar{\Gamma}$. 
Since $y_{k} \in \mathbf{R}^{n} \backslash \bar{\Gamma}, \underline{E}_{j}\left(x-y_{k}\right)$ is infinitely often differentiable with respect to $x$ in a neighborhood of $\bar{\Gamma}$. Noting that $\operatorname{supp} \underline{R^{\prime}} \underline{T} \subset \bar{\Gamma}$, we get

$$
\left\langle\left(\underline{R}\left(D^{\alpha} \underline{E}_{j}\left(x-y_{k}\right)\right)\right)^{\top}, \underline{T}(x)\right\rangle=\left\langle\left(D^{\alpha} \underline{E}_{j}\left(x-y_{k}\right)\right)^{\top},\left(\underline{R}^{\prime} \underline{T}\right)(x)\right\rangle .
$$

From (3), we conclude

$$
\left\langle\left(\left(D^{\alpha} \underline{E}_{j}\right)\left(x-y_{k}\right)\right)^{\top},\left(\underline{R}^{\prime} \underline{T}\right)(x)\right\rangle=0
$$

for $j=1, \ldots, N,|\alpha| \leq t+s_{j}-1$, and $k=1,2, \ldots$.

Step 3. Let $p>n$. Then the embedding

$$
W^{r_{0}+T+s, p}\left(\mathbf{R}^{n}\right)=W_{0}^{r_{0}+T+s, p}\left(\mathbf{R}^{n}\right) \hookrightarrow C^{0, r_{0}+T+s-1}\left(\mathbf{R}^{n}\right)
$$

is continous. Hence

$$
\left(C^{0, r_{0}+T+s-1}\left(\mathbf{R}^{n}\right)\right)^{\prime} \hookrightarrow\left(W_{0}^{r_{0}+T+s, p}\left(\mathbf{R}^{n}\right)\right)^{\prime}=W^{-r_{0}-T-s, p^{\prime}}\left(\mathbf{R}^{n}\right)
$$

where $p^{\prime}=\frac{p}{p-1}$ and

$$
\underline{R}^{\prime} \underline{T} \in W^{-r_{0}-T-s, p^{\prime}}\left(\mathbf{R}^{n}\right) .
$$

Since $\underline{L}$ is elliptic in the sense of Douglis-Nirenberg with $T=\left(t_{1}, \ldots, t_{N}\right)$ and $S=$ $\left(s_{1}, \ldots, s_{N}\right), \underline{L}^{*}$ is also elliptic in the sense of Douglis-Nirenberg, namely, with $T^{*}=$ $\left(s_{1}+t, \ldots, s_{N}+t\right)=S+t$ and $S^{*}=\left(t_{1}-t, \ldots, t_{N}-t\right)=T-t$. Hence, $-r_{0}-T-s=$ $-r_{0}-t-S^{*}-s$. Therefore

$$
\underline{R}^{\prime} \underline{T} \in W^{-r_{0}-t-S^{*}-s, p^{\prime}}\left(\mathbf{R}^{n}\right) .
$$

Since $\underline{R}^{\prime} \underline{T}$ has compact support, the convolution $\underline{E}^{*} *\left(\underline{R}^{\prime} \underline{T}\right)$ exists, and, by Lemma 2 ,

$$
\underline{E}^{*} *\left(\underline{R^{\prime}} \underline{T}\right) \in W_{l o c}^{-r_{0}-t+T^{*}-s, p^{\prime}}\left(\mathbf{R}^{n}\right)=W_{l o c}^{-r_{0}+S-s, p^{\prime}}\left(\mathbf{R}^{n}\right) .
$$

Now $\underline{L}^{*}\left(\underline{E}^{*} *\left(\underline{R}^{\prime} \underline{T}\right)\right)=\underline{R^{\prime}} \underline{T}$ and $\operatorname{supp} \underline{R^{\prime}} \underline{T} \subset \bar{\Gamma}$ imply $\underline{L}^{*}\left(\underline{E}^{*} *\left(\underline{R^{\prime}} \underline{T}\right)\right)=\underline{0}$ in $\mathbf{R}^{n} \backslash \bar{\Gamma}$. Thus, since $\underline{L}^{*}$ is elliptic, $\underline{E}^{*} *\left(\underline{R^{\prime}} \underline{T}\right) \in\left(C^{\infty}\left(\mathbf{R}^{n} \backslash \bar{\Gamma}\right)\right)^{N}$.

Step 4. If we note that $\underline{E}^{*}(x)=(\underline{E}(-x))^{\top}$, i.e., $E_{j i}^{*}(x)=E_{i j}(-x)$, we obtain for the $j$-th component of $\underline{E}^{*} *\left(\underline{R}^{\prime} \underline{T}\right)\left(\operatorname{let}\left(\underline{R^{\prime}} \underline{T}\right)^{i}\right.$ be the $i$-th component of $\left.\underline{R^{\prime}} \underline{T}\right)$

$$
\begin{aligned}
D^{\alpha}\left(\underline{E}^{*} *\left(\underline{R}^{\prime} \underline{T}\right)\right)^{j}\left(y_{k}\right) & =D^{\alpha}\left(\sum_{i=1}^{N} E_{j i}^{*} *\left(\underline{R}^{\prime} \underline{T}\right)^{i}\right)\left(y_{k}\right) \\
& =\sum_{i=1}^{N}\left(\left(D^{\alpha} E_{j i}^{*}\right) *\left(\underline{R^{\prime}} \underline{T}\right)^{i}\right)\left(y_{k}\right) \\
& =\sum_{i=1}^{N}\left\langle\left(D^{\alpha} E_{j i}^{*}\right)\left(y_{k}-x\right),\left(\underline{R}^{\prime} \underline{T}\right)^{i}(x)\right\rangle \\
& =(-1)^{|\alpha|} \sum_{i=1}^{N}\left\langle\left(D^{\alpha} E_{i j}\right)\left(x-y_{k}\right),\left(\underline{R}^{\prime} \underline{T}\right)^{i}(x)\right\rangle \\
& =(-1)^{|\alpha|}\left\langle\left(\left(D^{\alpha} \underline{E}_{j}\right)\left(x-y_{k}\right)\right)^{\top},\left(\underline{R}^{\prime} \underline{T}\right)(x)\right\rangle=0
\end{aligned}
$$

for $j=1, \ldots, N,|\alpha| \leq t+s_{j}-1$, and $k=1,2, \ldots$ The last equality follows from (4). For the third equality, i.e., for the expression of the convolution as the application of a functional, we used that $E_{j i}^{*}\left(y_{k}-x\right)$ is infinitely often differentiable with respect to $x$ in a neighborhood of the support of $\underline{R^{\prime}} \underline{T}$. 
Since $\underline{E}^{*} *\left(\underline{R}^{\prime} \underline{T}\right)$ is infinitely often differentiable in a neighborhood of $K \subset \mathbf{R}^{n} \backslash \bar{\Gamma}$, and since by assumption the sequence of points $\left(y_{k}\right)_{k=1}^{\infty}$ is dense in $K$,

$$
\left.D^{\alpha}\left(\underline{E}^{*} *\left(\underline{R}^{\prime} \underline{T}\right)\right)^{j}\right|_{K}=0
$$

for $j=1, \ldots, N$ and all $\alpha$ such that $|\alpha| \leq t+s_{j}-1$. Further, $\underline{L}^{*}\left(\underline{E}^{*} *\left(\underline{R}^{\prime} \underline{T}\right)\right)=\underline{0}$ in the connected set $\mathbf{R}^{n} \backslash \bar{\Gamma}$. Therefore, $\underline{w} \stackrel{\text { def }}{=} \underline{E}^{*} *\left(\underline{R^{\prime}} \underline{T}\right)$ is a solution to the following Cauchy problem:

$$
\begin{aligned}
\underline{L}^{*} \underline{w}=\underline{0} & \text { in } \mathbf{R}^{n} \backslash \bar{\Gamma} \\
\left.D^{\alpha} w^{j}\right|_{K}=0 & \text { for } j=1, \ldots, N, \quad|\alpha| \leq t+s_{j}-1=t_{j}^{*}-1 .
\end{aligned}
$$

By Lemma 3 , this problem only has the solution $\underline{w}=\underline{0}$. Hence, we obtain $\underline{E}^{*} *\left(\underline{R}^{\prime} \underline{T}\right) \equiv$ $\underline{0}$ in $\mathbf{R}^{n} \backslash \bar{\Gamma}$. Hence,

$$
\operatorname{supp}\left(\underline{E}^{*} *\left(\underline{R}^{\prime} \underline{T}\right)\right) \subset \bar{\Gamma} \quad \text { and } \quad \underline{E}^{*} *\left(\underline{R}^{\prime} \underline{T}\right) \in W_{l o c}^{-r_{0}+S-s, p^{\prime}}\left(\mathbf{R}^{n}\right) .
$$

By the choice of $r_{0}$, we get $-r_{0}+s_{i}-s \leq-r_{0}-s \leq(s-1)-s=-1$. From (5), one easily concludes $\underline{E}^{*} *\left(\underline{R}^{\prime} \underline{T}\right) \in\left(C^{0, r_{0}-S+s}\left(\mathbf{R}^{n}\right)\right)^{\prime}$. Hence, since $\operatorname{supp}\left(\underline{E}^{*} *\left(\underline{R}^{\prime} \underline{T}\right)\right) \subset \bar{\Gamma}$, we obtain

$$
\left\langle\underline{f}^{\top}, \underline{E}^{*} *\left(\underline{R}^{\prime} \underline{T}\right)\right\rangle=0
$$

for all $\underline{f} \in C^{0, r_{0}-S+s}\left(\mathbf{R}^{n} \backslash \bar{\Gamma}\right)$.

Step 5. We define the restriction operator $\underline{R}_{r_{0}-S+s}$ by

$$
\left.\underline{R}_{r_{0}-S+s} \underline{f}=\left(\left(\left.D_{\underline{v}}^{\beta^{\prime \prime}} f^{1}\right|_{\Gamma}\right)_{\left|\beta^{\prime \prime}\right| \leq r_{0}-s_{1}+s}, \ldots,\left(D_{\underline{v}}^{\beta^{\prime \prime}} f^{N} \mid \Gamma\right)_{\left|\beta^{\prime \prime}\right| \leq r_{0}-s_{N}+s}\right)\right)^{\top}
$$

for $\underline{f} \in C^{0, r_{0}-S+s}\left(\mathbf{R}^{n}\right)$. From Lemma 1, we get

$$
C^{0, r_{0}-S+s}\left(\mathbf{R}^{n} \backslash \bar{\Gamma}\right)=\left\{\underline{f} \in C^{0, r_{0}-S+s}\left(\mathbf{R}^{n}\right) \mid \underline{R}_{r_{0}-S+s} \underline{f}=\underline{0}\right\} .
$$

Hence,

$$
\left\langle\underline{f}^{\top}, \underline{E}^{*} *\left(\underline{R}^{\prime} \underline{T}\right)\right\rangle=0
$$

for all $\underline{f} \in C^{0, r_{0}-S+s}\left(\mathbf{R}^{n}\right)$ such that $\underline{R}_{r_{0}-S+s} \underline{f}=\underline{0}$.

Step 6 Now we want to compute $\left\langle\underline{f}^{\top}, \underline{E}^{*} *\left(\underline{R^{\prime}} \underline{T}\right)\right\rangle$ for arbitrary vector functions $\underline{f} \in C^{0, r_{0}-S+s}\left(\mathbf{R}^{n}\right)$. We define

$$
\operatorname{ker} \underline{R}_{r_{0}-S+s}=\left\{\underline{f} \in C^{0, r_{0}-S+s}\left(\mathbf{R}^{n}\right) \mid \underline{R}_{r_{0}-S+s} \underline{f}=\underline{0}\right\}
$$

and

$$
\begin{aligned}
\left(\operatorname{ker} \underline{R}_{r_{0}-S+s}\right)^{\perp}=\left\{\underline{F} \in\left(C^{0, r_{0}-S+s}\left(\mathbf{R}^{n}\right)\right)^{\prime} \mid\left\langle\underline{f}^{\top}, \underline{F}\right\rangle=0\right. \text { for all } \\
\\
\left.\underline{f} \in C^{0, r_{0}-S+s}\left(\mathbf{R}^{n}\right) \text { such that } \underline{R}_{r_{0}-S+s} \underline{f}=\underline{0}\right\} .
\end{aligned}
$$

Hence, (6) implies

$$
\underline{E}^{*} *\left(\underline{R^{\prime}} \underline{T}\right) \in\left(\operatorname{ker} \underline{R}_{r_{0}-S+s}\right)^{\perp} .
$$

Furthermore,

$$
\underline{R}_{r_{0}-S+s} \in L\left(C^{0, r_{0}-S+s}\left(\mathbf{R}^{n}\right), \prod_{i=1}^{N} \prod_{\left|\beta^{\prime \prime}\right| \leq r_{0}-s_{i}+s} C^{r_{0}-s_{i}+s-\left|\beta^{\prime \prime}\right|}(\bar{\Gamma})\right)
$$


and

$$
\underline{R}_{r_{0}-S+s}^{\prime} \in L\left(\prod_{i=1}^{N} \prod_{\left|\beta^{\prime \prime}\right| \leq r_{0}-s_{i}+s}\left(C^{r_{0}-s_{i}+s-\left|\beta^{\prime \prime}\right|}(\bar{\Gamma})\right)^{\prime},\left(C^{0, r_{0}-S+s}\left(\mathbf{R}^{n}\right)\right)^{\prime}\right) .
$$

Lemma 4 implies

$$
\operatorname{Im} \underline{R}_{r_{0}-S+s}=\prod_{i=1}^{N} \prod_{\left|\beta^{\prime \prime}\right| \leq r_{0}-s_{i}+s} C^{r_{0}-s_{i}+s-\left|\beta^{\prime \prime}\right|}(\bar{\Gamma})
$$

since, if $\underline{g}=\left(\left(g_{\beta^{\prime \prime}}^{1}\right)_{\left|\beta^{\prime \prime}\right| \leq r_{0}-s_{1}+s}, \ldots,\left(g_{\beta^{\prime \prime}}^{i}\right)_{\left|\beta^{\prime \prime}\right| \leq r_{0}-s_{i}+s}, \ldots,\left(g_{\beta^{\prime \prime}}^{N}\right)_{\left|\beta^{\prime \prime}\right| \leq r_{0}-s_{N}+s}\right)^{\top}$ is an arbitrary element of $\prod_{i=1}^{N} \prod_{\left|\beta^{\prime \prime}\right| \leq r_{0}-s_{i}+s} C^{r_{0}-s_{i}+s-\left|\beta^{\prime \prime}\right|}(\bar{\Gamma})$, then, by Lemma 4 , there exists for each $i=1, \ldots, N$, a function $h^{i} \in C^{0, r_{0}-s_{i}+s}\left(\mathbf{R}^{n}\right)$ such that $\left.D_{\underline{v}}^{\beta^{\prime \prime}} h^{i}\right|_{\Gamma}=g_{\beta^{\prime \prime}}^{i}$ for $\left|\beta^{\prime \prime}\right| \leq r_{0}-s_{i}+s$.

From the Closed Range Theorem (see [19], p.144), we get that $\operatorname{Im} \underline{R}_{r_{0}-S+s}^{\prime}=$ $\left(\operatorname{ker} \underline{R}_{r_{0}-S+s}\right)^{\perp}$. Further, from the Open Mapping Theorem and the Closed Range Theorem (see [19], p.147), it follows that

$$
\left(\underline{R}_{r_{0}-S+s}^{\prime}\right)^{-1} \in L\left(\operatorname{Im} \underline{R}_{r_{0}-S+s}^{\prime}, \prod_{i=1}^{N} \prod_{\left|\beta^{\prime \prime}\right| \leq r_{0}-s_{i}+s}\left(C^{r_{0}-s_{i}+s-\left|\beta^{\prime \prime}\right|}(\bar{\Gamma})\right)^{\prime}\right),
$$

and hence that

$$
\left(\underline{R}_{r_{0}-S+s}^{\prime}\right)^{-1} \in L\left(\left(\operatorname{ker} \underline{R}_{r_{0}-S+s}\right)^{\perp}, \prod_{i=1}^{N} \prod_{\left|\beta^{\prime \prime}\right| \leq r_{0}-s_{i}+s}\left(C^{r_{0}-s_{i}+s-\left|\beta^{\prime \prime}\right|}(\bar{\Gamma})\right)^{\prime}\right) .
$$

Since $\underline{E}^{*} *\left(\underline{R}^{\prime} \underline{T}\right) \in\left(\operatorname{ker} \underline{R}_{r_{0}-S+s}\right)^{\perp}$, the equation

$$
\underline{R}_{r_{0}-S+s}^{\prime} \underline{\Lambda}=\underline{E}^{*} *\left(\underline{R}^{\prime} \underline{T}\right)
$$

has a unique solution

$$
\begin{aligned}
\underline{\Lambda} & =\left(\left(\lambda_{1, \beta^{\prime \prime}}\right)_{\left|\beta^{\prime \prime}\right| \leq r_{0}-s_{1}+s}, \ldots,\left(\lambda_{i, \beta^{\prime \prime}}\right)_{\left|\beta^{\prime \prime}\right| \leq r_{0}-s_{i}+s}, \ldots,\left(\lambda_{N, \beta^{\prime \prime}}\right)_{\left|\beta^{\prime \prime}\right| \leq r_{0}-s_{N}+s}\right)^{\top} \\
& \in \prod_{i=1}^{N}\left(\prod_{\left|\beta^{\prime \prime}\right| \leq r_{0}-s_{i}+s}\left(C^{r_{0}-s_{i}+s-\left|\beta^{\prime \prime}\right|}(\bar{\Gamma})\right)^{\prime}\right) .
\end{aligned}
$$

Hence, for all $\underline{h} \in C^{0, r_{0}-S+s}\left(\mathbf{R}^{n}\right)$,

$$
\begin{aligned}
\left\langle\underline{h}^{\top}, \underline{E}^{*} *\left(\underline{R}^{\prime} \underline{T}\right)\right\rangle & =\left\langle\underline{h}^{\top}, \underline{R}_{r_{0}-S+s}^{\prime} \underline{\Lambda}\right\rangle=\left\langle\left(\underline{R}_{r_{0}-S+s} \underline{h}\right)^{\top}, \underline{\Lambda}\right\rangle \\
& =\sum_{i=1}^{N} \sum_{\left|\beta^{\prime \prime}\right| \leq r_{0}-s_{i}+s}\left\langle\left. D_{\underline{v}}^{\beta^{\prime \prime}} h^{i}\right|_{\Gamma}, \lambda_{i, \beta^{\prime \prime}}\right\rangle .
\end{aligned}
$$

Step 7. Now let $\underline{u} \in C^{0, r_{0}+T+s}\left(\mathbf{R}^{n}\right)$ be an arbitrary vector function. Then $\underline{L u} \in$ $C^{0, r_{0}-S+s}\left(\mathbf{R}^{n}\right)$. We set $\underline{h}=\underline{L u}$ in $(7)$ to get

$$
\left\langle(\underline{L u})^{\top}, \underline{E}^{*} *\left(\underline{R^{\prime}} \underline{T}\right)\right\rangle=\sum_{i=1}^{N} \sum_{\left|\beta^{\prime \prime}\right| \leq r_{0}-s_{i}+s}\left\langle\left. D_{\underline{v}}^{\beta^{\prime \prime}}(\underline{L u})^{i}\right|_{\Gamma}, \lambda_{i, \beta^{\prime \prime}}\right\rangle
$$

for all $\underline{u} \in C^{0, r_{0}+T+s}\left(\mathbf{R}^{n}\right)$. From

$$
\left\langle(\underline{L u})^{\top}, \underline{E}^{*} *\left(\underline{R^{\prime}} \underline{T}\right)\right\rangle=\left\langle\underline{u}^{\top}, \underline{L}^{*}\left(\underline{E}^{*} *\left(\underline{R^{\prime}} \underline{T}\right)\right)\right\rangle=\left\langle\underline{u}^{\top}, \underline{R}^{\prime} \underline{T}\right\rangle=\left\langle(\underline{R u})^{\top}, \underline{T}\right\rangle
$$


and from

$$
\begin{aligned}
\sum_{i=1}^{N} & \sum_{\left|\beta^{\prime \prime}\right| \leq r_{0}-s_{i}+s}\left\langle\left. D_{\underline{v}}^{\beta^{\prime \prime}}(\underline{L u})^{i}\right|_{\Gamma}, \lambda_{i, \beta^{\prime \prime}}\right\rangle \\
= & \sum_{l=0}^{r_{0}} \sum_{i=i_{l}^{*}\left|\beta^{\prime \prime}\right|=r_{0}-s_{i}+s-l}^{N} \sum_{\underline{v}}\left\langle\left. D^{\beta^{\prime \prime}}(\underline{L u})^{i}\right|_{\Gamma}, \lambda_{i, \beta^{\prime \prime}}\right\rangle,
\end{aligned}
$$

we conclude

$$
\left\langle(\underline{R u})^{\top}, \underline{T}\right\rangle=\sum_{l=0}^{r_{0}} \sum_{i=i_{l}^{*}}^{N} \sum_{\left|\beta^{\prime \prime}\right|=r_{0}-s_{i}+s-l}\left\langle\left. D_{\underline{v}}^{\beta^{\prime \prime}}(\underline{L u})^{i}\right|_{\Gamma}, \lambda_{i, \beta^{\prime \prime}}\right\rangle
$$

for all $\underline{u} \in C^{0, r_{0}+T+s}\left(\mathbf{R}^{n}\right)$. Here $i_{l}^{*}$ is defined to be the smallest integer $i$ such that $r_{0}-s_{i}+s-l \geq 0$.

In the following steps, $\left.(\underline{L u})^{i}\right|_{\Gamma}$ will be represented by $D_{\underline{v}}^{\alpha}$ according to the considerations in Section 4.6:

$$
\begin{aligned}
\left.(\underline{L u})^{i}\right|_{\Gamma} & =\left.\sum_{j=1}^{N_{i}} \sum_{|\alpha| \leq s_{i}+t_{j}} a_{\alpha}^{i j} D^{\alpha} u^{j}\right|_{\Gamma} \\
& =\left.\sum_{j=1}^{N_{i}} \sum_{|\alpha| \leq s_{i}+t_{j}} a_{\alpha}^{i j}\left(\underline{W}(z) \cdot D_{\underline{v}(z)}\right)^{\alpha} u^{j}(x)\right|_{x=z \in \Gamma} \\
& =\left.\sum_{j=1}^{N_{i}} \sum_{|\alpha| \leq s_{i}+t_{j}} \tilde{a}_{\alpha}^{i j}(z) D_{\underline{v}(z)}^{\alpha} u^{j}(x)\right|_{x=z \in \Gamma}
\end{aligned}
$$

Here $N_{i}$ is the maximal integer $j$ such that $t_{j}+s_{i} \geq 0$.

Step 8. In the following steps, we will show that

$$
\lambda_{i, \beta^{\prime \prime}}=0 \text { for }\left|\beta^{\prime \prime}\right| \leq r_{0}-s_{i}+s \text { and } i=1, \ldots, N .
$$

We proceed as follows. Instead of (8), we investigate the equation

$$
\left\langle(\underline{R u})^{\top}, \underline{T}\right\rangle=\sum_{l=r}^{r_{0}} \sum_{i=i_{l}^{*}\left|\beta^{\prime \prime}\right|=r_{0}-s_{i}+s-l}^{N}\left\langle\left. D_{\underline{v}}^{\beta^{\prime \prime}}(\underline{L u})^{i}\right|_{\Gamma}, \lambda_{i, \beta^{\prime \prime}}\right\rangle
$$

for an arbitrary integer $r$ such that $0 \leq r \leq r_{0}$ and for all $\underline{u} \in C^{0, r_{0}+T+s}\left(\mathbf{R}^{n}\right)$. Then we show that

$$
\lambda_{i, \beta^{\prime \prime}}=0 \text { for }\left|\beta^{\prime \prime}\right|=r_{0}-s_{i}+s-r \quad \text { and } \quad i=i_{r}^{*}, \ldots, N .
$$

Hence we obtain from (9) the new equation

$$
\left\langle(\underline{R u})^{\top}, \underline{T}\right\rangle=\sum_{l=r+1}^{r_{0}} \sum_{i=i_{l}^{*}}^{N} \sum_{\left|\beta^{\prime \prime}\right|=r_{0}-s_{i}+s-l}\left\langle\left. D_{\underline{v}}^{{\beta^{\prime \prime}}^{\prime \prime}}(\underline{L u})^{i}\right|_{\Gamma}, \lambda_{i, \beta^{\prime \prime}}\right\rangle
$$

for all $\underline{u} \in C^{0, r_{0}+T+s}\left(\mathbf{R}^{n}\right)$ in the case $r<r_{0}$ and

$$
\left\langle(\underline{R u})^{\top}, \underline{T}\right\rangle=0
$$

for all $\underline{u} \in C^{0, r_{0}+T+s}\left(\mathbf{R}^{n}\right)$ in the case $r=r_{0}$.

If we apply the implication (9) $\Longrightarrow(10)$ for $r=0$, then for $r=1, \ldots, r=r_{0}$, then we finally obtain $\lambda_{i, \beta^{\prime \prime}}=0$ for all $\beta^{\prime \prime}$ such that $\left|\beta^{\prime \prime}\right| \leq r_{0}-s_{i}+s$ and all $i=1, \ldots, N$. 
The proof of (10) is by induction. To this end, we will introduce an order relation in Step 10 on the set of pairs $\left\{\left(i, \beta^{\prime \prime}\right)\left|i=i_{r}^{*}, \ldots, N ;\right| \beta^{\prime \prime} \mid=r_{0}-s_{i}+s-r\right\}$. Equation (9) is now the basis for the following steps.

Step 9. Now assume for an $r$ with $0 \leq r \leq r_{0}$ that

$$
\left\langle(\underline{R u})^{\top}, \underline{T}\right\rangle=\sum_{l=r}^{r_{0}} \sum_{i=i_{l}^{*}}^{N} \sum_{\left|\beta^{\prime \prime}\right|=r_{0}-s_{i}+s-l}\left\langle\left. D_{\underline{v}}^{\beta^{\prime \prime}}(\underline{L u})^{i}\right|_{\Gamma}, \lambda_{i, \beta^{\prime \prime}}\right\rangle
$$

for all $\underline{u} \in C^{0, r_{0}+T+s}\left(\mathbf{R}^{n}\right)$. In this step, we show that for all $\underline{u} \in C^{0, r_{0}+T+s}\left(\mathbf{R}^{n}\right)$ which satisfy

$$
\left.D_{\underline{v}}^{\gamma^{\prime \prime}} u^{j}\right|_{\Gamma}=0 \text { for }\left|\gamma^{\prime \prime}\right| \leq r_{0}+t_{j}+s-r-1 \quad \text { and } j=1, \ldots, N_{i_{r}^{*}}
$$

(if $r_{0}+t_{j}+s-r-1<0$ for some $j$ then the condition $\left.D_{\underline{v}}^{\gamma^{\prime \prime}} u^{j}\right|_{\Gamma}=0$ on the corresponding $u^{j}$ is dropped), we can conclude

$$
0=\sum_{i=i_{r}^{*}\left|\beta^{\prime \prime}\right|=r_{0}-s_{i}+s-r}^{N}\left\langle\left. D_{\underline{v}}^{\beta^{\prime \prime}}(\underline{L u})^{i}\right|_{\Gamma}, \lambda_{i, \beta^{\prime \prime}}\right\rangle .
$$

To this end, we first show $\underline{R u}=\underline{0}$ for these $\underline{u}$. We have

$$
\underline{R u}=\left(\left(\left.D_{\underline{v}}^{\alpha^{\prime \prime}} u^{1}\right|_{\Gamma}\right)_{\left|\alpha^{\prime \prime}\right| \leq t_{1}+s-1}, \ldots,\left(\left.D_{\underline{v}}^{\alpha^{\prime \prime}} u^{N^{*}}\right|_{\Gamma}\right)_{\left|\alpha^{\prime \prime}\right| \leq t_{N^{*}+s-1}}\right)^{\top}
$$

where $N^{*}$ is the maximal integer $j$ with $t_{j}+s-1 \geq 0$. For $j \leq N^{*}$, we have $0 \leq t_{j}+s-1<t_{j}+s \leq t_{j}+s_{i_{r}^{*}}$. Since $N_{i_{r}^{*}}$ is defined to be the maximal integer $j$ with $t_{j}+s_{i_{r}^{*}} \geq 0$, we get $N_{i_{r}^{*}} \geq N^{*}$. Further, since $r \leq r_{0}$, we obtain $r_{0}+t_{j}+s-r-1 \geq$ $t_{j}+s-1 \geq 0$ for all $j=1, \ldots, N^{*}$. Hence, $\left.D_{\underline{v}}^{\alpha^{\prime \prime}} u^{j}\right|_{\Gamma}=0$ for $|\alpha| \leq t_{j}+s-1$ and $j=1, \ldots, N^{*}$. Thus $\underline{R u}=\underline{0}$. Hence, we get

$$
0=\sum_{l=r}^{r_{0}} \sum_{i=i_{l}^{*}}^{N} \sum_{\left|\beta^{\prime \prime}\right|=r_{0}-s_{i}+s-l}\left\langle\left. D_{\underline{v}}^{\beta^{\prime \prime}}(\underline{L u})^{i}\right|_{\Gamma}, \lambda_{i, \beta^{\prime \prime}}\right\rangle
$$

for $\underline{u}$ with the property (11). Thus, we showed (12) already for the case $r=r_{0}$. It remains to investigate the case $r<r_{0}$. For $r<r_{0}$, we obtain

$$
\begin{aligned}
& \sum_{l=r}^{r_{0}} \sum_{i=i_{l}^{*}\left|\beta^{\prime \prime}\right|=r_{0}-s_{i}+s-l}^{N}\left\langle\left. D_{\underline{v}}^{\beta^{\prime \prime}}(\underline{L u})^{i}\right|_{\Gamma}, \lambda_{i, \beta^{\prime \prime}}\right\rangle \\
& =\sum_{i=i_{r}^{*}\left|\beta^{\prime \prime}\right|=r_{0}-s_{i}+s-r} \sum_{\underline{v}}\left\langle\left. D_{\underline{\beta^{\prime \prime}}}(\underline{L u})^{i}\right|_{\Gamma}, \lambda_{i, \beta^{\prime \prime}}\right\rangle \\
& \quad+\sum_{i=i_{r+1}^{*}\left|\beta^{\prime \prime}\right| \leq r_{0}-s_{i}+s-r-1}^{N}\left\langle\left. D_{\underline{\underline{\beta^{\prime \prime}}}}(\underline{L u})^{i}\right|_{\Gamma}, \lambda_{i, \beta^{\prime \prime}}\right\rangle .
\end{aligned}
$$

We prove $\left.D_{\underline{v}}^{\beta^{\prime \prime}}(\underline{L u})^{i}\right|_{\Gamma}=0$ for $\left|\beta^{\prime \prime}\right| \leq r_{0}-s_{i}+s-r-1$ and $i_{r+1}^{*} \leq i \leq N$. From $r_{0}-s_{i_{r+1}^{*}}+s-r-1 \geq 0$ and $s_{i_{r+1}^{*}}+t_{j} \geq 0$ for $j=1, \ldots, N_{i_{r+1}^{*}}$, we conclude $r_{0}+t_{j}+s-r-1 \geq 0$ for $j=1, \ldots, N_{i_{r+1}^{*}}$. Since $N_{i_{r+1}^{*}} \leq N_{i_{r}^{*}},\left.D_{\underline{v}}^{\gamma^{\prime \prime}} u^{j}\right|_{\Gamma}=0$ for all $\gamma^{\prime \prime}$ such that $\left|\gamma^{\prime \prime}\right| \leq r_{0}+t_{j}+s-r-1$ and $j=1, \ldots, N_{i_{r+1}^{*}}$. Hence, $\left.D_{\underline{v}}^{\gamma} u^{j}\right|_{\Gamma}=0$ for all multi-indices $\gamma=\left(\gamma_{1}, \ldots, \gamma_{n}\right)$ such that $|\gamma| \leq r_{0}+t_{j}+s-r-1$ and $\bar{j}=1, \ldots, N_{i_{r+1}^{*}}$. 
For arbitrary multi-indices $\beta^{\prime \prime}=\left(0, \ldots, 0, \beta_{d+1}, \ldots, \beta_{n}\right)$ and $i=1, \ldots, N$, we have

$$
\left.D_{\underline{v}}^{\beta^{\prime \prime}}(\underline{L u})^{i}\right|_{\Gamma}=\left.\sum_{j=1}^{N_{i}} \sum_{|\alpha| \leq s_{i}+t_{j}} \tilde{a}_{\alpha}^{i j}(z) \cdot D_{\underline{v}(z)}^{\alpha+\beta^{\prime \prime}} u^{j}(x)\right|_{x=z \in \Gamma} .
$$

For $i \geq i_{r+1}^{*}, j \leq N_{i} \leq N_{i_{r+1}^{*}},\left|\beta^{\prime \prime}\right| \leq r_{0}-s_{i}+s-r-1$, and $|\alpha| \leq s_{i}+t_{j}$, we have $\left|\alpha+\beta^{\prime \prime}\right| \leq r_{0}+t_{j}+s-r-1$, and hence $\left.D_{\underline{v}}^{\alpha+\beta^{\prime \prime}} u^{j}\right|_{\Gamma}=0$. Now (15) implies $\left.D_{\underline{v}}^{\beta^{\prime \prime}}(\underline{L u})^{i}\right|_{\Gamma}=0$ for $i \geq i_{r+1}^{*}$ and $\left|\beta^{\prime \prime}\right| \leq r_{0}-s_{i}+s-r-1$. Hence, the second summand on the right-hand side of (14) vanishes. Thus, by (13), equation (12) is also true for $r<r_{0}$.

By an appropriate choice of $\left.D_{\underline{v}}^{\gamma^{\prime \prime}} u^{j}\right|_{\Gamma}$ for $\left|\gamma^{\prime \prime}\right|=r_{0}+t_{j}+s-r$, we will show inductively that $\lambda_{i, \beta^{\prime \prime}}=0$ for $\left|\beta^{\prime \prime}\right| \stackrel{\underline{v}}{=} r_{0}-s_{i}+s-r$ and $i=i_{r}^{*}, \ldots, N$.

Step 10. For this proof by induction, we introduce the following (lexicographical) order relation on the set of all multi-indices: for $\alpha \neq \beta\left(\alpha=\left(\alpha_{1}, \ldots, \alpha_{n}\right), \beta=\left(\beta_{1}, \ldots, \beta_{n}\right)\right)$, let

$$
\begin{array}{lll}
\alpha<\beta \quad \text { if } 1 . \alpha_{1}<\beta_{1} & \\
& \text { or } 2 . \alpha_{k+1}<\beta_{k+1} & \text { and } \alpha_{l}=\beta_{l} \\
& & \text { for } l=1, \ldots, k \text { for some } k<n .
\end{array}
$$

Furthermore, we introduce an order relation on the set of pairs $\left(i, \beta^{\prime \prime}\right)$ with $i_{r}^{*} \leq i \leq N$ and $\left|\beta^{\prime \prime}\right|=r_{0}-s_{i}+s-r$. We set

$$
M_{r}=\left\{\left(i, \beta^{\prime \prime}\right)\left|i=i_{r}^{*}, \ldots, N ; \quad\right| \beta^{\prime \prime} \mid=r_{0}-s_{i}+s-r\right\} .
$$

For $\left(i, \alpha^{\prime \prime}\right),\left(j, \beta^{\prime \prime}\right) \in M_{r}$, let

$$
\begin{array}{ll}
\left(i, \alpha^{\prime \prime}\right)<\left(j, \beta^{\prime \prime}\right) \quad \text { if } 1 . \alpha^{\prime \prime}<\beta^{\prime \prime} \\
& \text { or } 2 . i<j \text { and } \alpha^{\prime \prime}=\beta^{\prime \prime} .
\end{array}
$$

We order the elements according to this order relation and denote them by $\left(i_{k}, \beta_{(k)}^{\prime \prime}\right)$ where $k=1, \ldots, k_{1}$ and $k_{1}$ is the number of elements in $M_{r}$. Hence, $\left(i_{k}, \beta_{(k)}^{\prime \prime}\right)<$ $\left(i_{k+1}, \beta_{(k+1)}^{\prime \prime}\right)$ for $k=1, \ldots, k_{1}-1$.

Step 11. We show that $\lambda_{i_{1}, \beta_{(1)}^{\prime \prime}}=0$. Clearly, $i_{1}=i_{r}^{*}$ and $\beta_{(1)}^{\prime \prime}=\left(0, \ldots, 0, r_{0}-s_{i_{1}}+s-r\right)$. From $r_{0}-s_{i_{1}}+s-r \geq 0$ and $s_{i_{1}}+t_{j} \geq 0$ for $j=1, \ldots, N_{i_{1}}$, we conclude $r_{0}+t_{j}+s-r \geq 0$ for $j \leq N_{i_{1}}$.

For an arbitrary function $g \in C^{r}(\bar{\Gamma})$, let $\underline{u} \in C_{0}^{r_{0}+T+s}\left(\mathbf{R}^{n}\right)$ be a vector function that satisfies the following properties:

$$
\begin{gathered}
\left.\sum_{j=1}^{N_{i}} \tilde{a}_{\left(0, \ldots, 0, s_{i}+t_{j}\right)}^{i j}(z) \cdot D_{\underline{v}(z)}^{\left(0, \ldots, r_{0}+t_{j}+s-r\right)} u^{j}(x)\right|_{x=z \in \Gamma} \\
=\left\{\begin{array}{ll}
g & \text { for } i=i_{1} \\
0 & \text { for } i \neq i_{1}
\end{array}\left(i=i_{1}, \ldots, N\right),\right.
\end{gathered}
$$

2. $\left.D_{\underline{v}}^{\gamma^{\prime \prime}} u^{j}\right|_{\Gamma}=0$ for $\left|\gamma^{\prime \prime}\right| \leq r_{0}+t_{j}+s-r, \gamma^{\prime \prime} \neq\left(0, \ldots, 0, r_{0}+t_{j}+s-r\right)$,

$$
j=1, \ldots, N_{i_{1}} \text {. }
$$

(If $N_{i_{1}}<N$, the functions $u^{j}$ for $N_{i_{1}}<j \leq N$ can be arbitrary.) The existence of such vector functions $\underline{u}$ follows from Lemma 5 with $i_{0}=i_{1}$ and $\beta_{(0)}^{\prime \prime}=\left(0, \ldots, 0, r_{0}-\right.$ 
$\left.s_{i_{1}}+s-r\right)$. From (17), we get

$$
\begin{aligned}
& \left.D_{\underline{v}}^{\gamma} u^{j}\right|_{\Gamma}=0 \text { for all } \gamma=\left(\gamma_{1}, \ldots, \gamma_{n}\right) \text { with }|\gamma| \leq r_{0}+t_{j}+s-r, \\
& \gamma \neq\left(0, \ldots, 0, r_{0}+t_{j}+s-r\right), \quad \text { and } j=1, \ldots, N_{i_{1}} .
\end{aligned}
$$

From condition (17), we concluded in Step 9 that

$$
\sum_{i=i_{1}}^{N} \sum_{\left|\beta^{\prime \prime}\right|=r_{0}-s_{i}+s-r}\left\langle\left. D_{\underline{v}}^{\beta^{\prime \prime}}(\underline{L u})^{i}\right|_{\Gamma}, \lambda_{i, \beta^{\prime \prime}}\right\rangle=0 .
$$

For vector functions $\underline{u}$ with the properties (16) and (17), we will show that

$$
\sum_{i=i_{1}}^{N} \sum_{\left|\beta^{\prime \prime}\right|=r_{0}-s_{i}+s-r}\left\langle\left. D_{\underline{v}}^{\beta^{\prime \prime}}(\underline{L u})^{i}\right|_{\Gamma}, \lambda_{i, \beta^{\prime \prime}}\right\rangle=\left\langle g, \lambda_{i_{1}, \beta_{(1)}^{\prime \prime}}\right\rangle .
$$

To this end, we investigate three cases.

Case 1. Let $i=i_{1}$ and $\beta^{\prime \prime}=\beta_{(1)}^{\prime \prime}=\left(0, \ldots, 0, r_{0}+s_{i_{1}}+s-r\right)$. Then $\alpha+\beta_{(1)}^{\prime \prime}=$ $\left(0, \ldots, 0, r_{0}+t_{j}+s-r\right)$ only for $\alpha=\left(0, \ldots, 0, s_{i_{1}}+t_{j}\right)$. By (18),

$$
\begin{aligned}
\left.D_{\underline{v}}^{\beta_{(1)}^{\prime \prime}}(\underline{L u})^{i_{1}}\right|_{\Gamma} & =\left.\sum_{j=1}^{N_{i_{1}}} \sum_{|\alpha| \leq s_{i_{1}}+t_{j}} \tilde{a}_{\alpha}^{i_{1} j}(z) \cdot D_{\underline{v}(z)}^{\alpha+\beta_{(1)}^{\prime \prime}} u^{j}(x)\right|_{x=z \in \Gamma} \\
& =\left.\sum_{j=1}^{N_{i_{1}}} \tilde{a}_{\left(0, \ldots, 0, s_{i_{1}}+t_{j}\right)}^{i_{1} j}(z) \cdot D_{\underline{v}(z)}^{\left(0, \ldots, 0, r_{0}+t_{j}+s-r\right)} u^{j}(x)\right|_{x=z \in \Gamma}
\end{aligned}
$$

and then, by (16),

$$
\left.D_{\underline{v}}^{\beta_{(1)}^{\prime \prime}}(\underline{L u})^{i_{1}}\right|_{\Gamma}=g .
$$

Case 2. Let $i>i_{1}$ and $\beta^{\prime \prime}=\left(0, \ldots, 0, r_{0}-s_{i}+s-r\right)$. Then $\alpha+\beta^{\prime \prime}=\left(0, \ldots, 0, r_{0}+\right.$ $\left.t_{j}+s-r\right)$ only for $\alpha=\left(0, \ldots, 0, s_{i}+t_{j}\right)$. From (18) and (16), we get

$$
\left.D_{\underline{v}}^{\beta^{\prime \prime}}(\underline{L u})^{i}\right|_{\Gamma}=0 .
$$

Case 3. Let $i\left(i_{1} \leq i \leq N\right)$ be arbitrary and let $\beta^{\prime \prime}$ be a multi-index with $\left|\beta^{\prime \prime}\right| \leq$ $r_{0}-s_{i}+s-r$ and $\beta^{\prime \prime} \neq\left(0, \ldots, 0, r_{0}-s_{i}+s-r\right)$; then $\alpha+\beta^{\prime \prime} \neq\left(0, \ldots, 0, r_{0}+t_{j}+s-r\right)$ for all $\alpha$ with $|\alpha| \leq s_{i}+t_{j}$. Hence, $\left.D_{\underline{v}}^{\alpha+\beta^{\prime \prime}} u^{j}\right|_{\Gamma}=0$ for $j=1, \ldots, N_{i} \leq N_{i_{1}}$, and thus

$$
\left.D_{\underline{v}}^{\beta^{\prime \prime}}(\underline{L u})^{i}\right|_{\Gamma}=\left.\sum_{j=1}^{N_{i}} \sum_{|\alpha| \leq s_{i}+t_{j}} \tilde{a}_{\alpha}^{i j}(z) \cdot D_{\underline{v}(z)}^{\alpha+\beta^{\prime \prime}} u^{j}(x)\right|_{x=z \in \Gamma}=0 .
$$

Hence, (20) is proved. Thus, by (19),

$$
\left\langle g, \lambda_{i_{1}, \beta_{(1)}^{\prime \prime}}\right\rangle=0 \text {. }
$$

Since $\lambda_{i_{1}, \beta_{(1)}^{\prime \prime}} \in\left(C^{r}(\bar{\Gamma})\right)^{\prime}$ and since $g \in C^{r}(\bar{\Gamma})$ is an arbitrary function, we obtain $\lambda_{i_{1}, \beta_{(1)}^{\prime \prime}}=0$.

Step 12. For the proof by induction, we now may assume that

$$
\lambda_{i_{l}, \beta_{(l)}^{\prime \prime}}=0 \text { for } l=1, \ldots, k-1
$$

for some $k$ with $2 \leq k \leq k_{1}$. We will conclude that $\lambda_{i_{k}, \beta_{(k)}^{\prime \prime}}=0$. Then we will have proved $\lambda_{i_{l}, \beta_{(l)}^{\prime \prime}}=0$ for all $l=1, \ldots, k_{1}$. 
So let $\left(i_{k}, \beta_{(k)}^{\prime \prime}\right)$ be the $k$-th element of $M_{r}$. Then $\left|\beta_{(k)}^{\prime \prime}\right|=r_{0}-s_{i_{k}}+s-r$ for $\beta_{(k)}^{\prime \prime}=\left(0, \ldots, 0, \beta_{d+1}^{(k)}, \ldots, \beta_{n}^{(k)}\right)$.

By $i^{*}$, we denote the smallest integer $i$ with $\beta_{n}^{(k)}+s_{i_{k}}-s_{i} \geq 0$ and $i_{r}^{*} \leq i \leq N$. For $j \leq N_{i^{*}}$, we have $s_{i^{*}}+t_{j} \geq 0$ and hence $\beta_{n}^{(k)}+s_{i_{k}}+t_{j} \geq 0$. Furthermore, $i^{*} \leq i_{k} \leq N$.

For an arbitrary function $g \in C^{r}(\bar{\Gamma})$, let $\underline{u} \in C_{0}^{r_{0}+T+s}\left(\mathbf{R}^{n}\right)$ be a vector function with the following properties:

1. $\left.\sum_{j=1}^{N_{i}} \tilde{a}_{\left(0, \ldots, 0, s_{i}+t_{j}\right)}^{i j}(z) D_{\underline{v}(z)}^{\left(0, \ldots, 0, \beta_{d+1}^{(k)}, \ldots, \beta_{n-1}^{(k)}, \beta_{n}^{(k)}+s_{i_{k}}+t_{j}\right)} u^{j}(x)\right|_{x=z \in \Gamma}$

$$
=\left\{\begin{array}{ll}
g & \text { for } i=i_{k} \\
0 & \text { for } i \neq i_{k}
\end{array}\left(i=i^{*}, \ldots, N\right),\right.
$$

2. $\left.\quad D_{\underline{v}}^{\gamma^{\prime \prime}} u^{j}\right|_{\Gamma}=0$ for $\gamma^{\prime \prime}$ with $\left|\gamma^{\prime \prime}\right| \leq r_{0}+t_{j}+s-r \quad$ and

$$
\gamma^{\prime \prime} \neq\left(0, \ldots, 0, \beta_{d+1}^{(k)}, \ldots, \beta_{n-1}^{(k)}, \beta_{n}^{(k)}+s_{i_{k}}+t_{j}\right) \text { and } j=1, \ldots, N_{i^{*}},
$$

3. $u^{j} \equiv 0$ for $N_{i^{*}}<j \leq N_{i_{r}^{*}}$, if $N_{i^{*}}<N_{i_{r}^{*}}$.

(If $N_{i_{r}^{*}}<N$, the $u^{j}$ can be arbitrary for $N_{i_{r}^{*}}<j \leq N$.)

The existence of such a vector function follows from Lemma 5 with $i_{0}=i_{k}$ and $\beta_{(0)}^{\prime \prime}=\beta_{(k)}^{\prime \prime}$.

From (23), we obtain

$$
\begin{aligned}
& \left.D_{\underline{v}}^{\gamma} u^{j}\right|_{\Gamma}=0 \text { for all } \gamma=\left(\gamma_{1}, \ldots, \gamma_{n}\right) \text { with }|\gamma| \leq r_{0}+t_{j}+s-r \\
& \text { and } \gamma \neq\left(0, \ldots, 0, \beta_{d+1}^{(k)}, \ldots, \beta_{n-1}^{(k)}, \beta_{n}^{(k)}+s_{i_{k}}+t_{j}\right) \text { and } j=1, \ldots, N_{i^{*}} \text {, }
\end{aligned}
$$

From (23) and (24), we concluded in Step 9 that

$$
\sum_{i=i_{r}^{*}\left|\beta^{\prime \prime}\right|=r_{0}-s_{i}+s-r}^{N}\left\langle\left. D_{\underline{v}}^{\beta^{\prime \prime}}(\underline{L u})^{i}\right|_{\Gamma}, \lambda_{i, \beta^{\prime \prime}}\right\rangle=0 .
$$

In the next step, we will show that for vector functions $\underline{u}$ with the properties (22), (23), and (24), the following holds

$$
\sum_{i=i_{r}^{*}\left|\beta^{\prime \prime}\right|=r_{0}-s_{i}+s-r}^{N}\left\langle\left. D_{\underline{v}}^{\beta^{\prime \prime}}(\underline{L u})^{i}\right|_{\Gamma}, \lambda_{i, \beta^{\prime \prime}}\right\rangle=\left\langle g, \lambda_{i_{k}, \beta_{(k)}^{\prime \prime}}\right\rangle
$$

Then, with one exception, all summands vanish. Either $\left.D_{\underline{v}}^{\beta^{\prime \prime}}(\underline{L u})^{i}\right|_{\Gamma}=0$ or from the induction hypothesis (21), we have $\lambda_{i, \beta^{\prime \prime}}=0$.

Step 13. We will now prove (27). We consider four cases. Let $i_{r}^{*} \leq i \leq N$ and let $\underline{u} \in C_{0}^{r_{0}+T+s}\left(\mathbf{R}^{n}\right)$ be a vector function with the properties (22), (23), and (24). The $u^{j}$ for $j>N_{i_{r}^{*}}$ can be arbitrary-they are not considered by $(\underline{L u})^{i}$ for $i \geq i_{r}^{*}$. 
Case 1. Let $i=i_{k}$ and $\beta^{\prime \prime}=\beta_{(k)}^{\prime \prime}$. Only if $\alpha=\left(0, \ldots, 0, s_{i_{k}}+t_{j}\right)$ do we obtain $\alpha+\beta_{(k)}^{\prime \prime}=\left(0, \ldots, 0, \beta_{d+1}^{(k)}, \ldots, \beta_{n-1}^{(k)}, \beta_{n}^{(k)}+s_{i_{k}}+t_{j}\right)$. From (25), we get

$$
\begin{aligned}
\left.D_{\underline{v}}^{\beta_{(k)}^{\prime \prime}}(\underline{L u})^{i_{k}}\right|_{\Gamma} & =\left.\sum_{j=1}^{N_{i_{k}}} \sum_{|\alpha| \leq s_{i_{k}}+t_{j}} \tilde{a}_{\alpha}^{i_{k} j}(z) \cdot D_{\underline{v}(z)}^{\alpha+\beta_{(k)}^{\prime \prime}} u^{j}(x)\right|_{x=z \in \Gamma} \\
& =\sum_{j=1}^{N_{i_{k}}} \tilde{a}_{\left(0, \ldots, 0, s_{i_{k}}+t_{j}\right)}^{i_{k} j}(z) \cdot D_{\underline{v}(z)}^{\left(0, \ldots, 0, s_{i_{k}}+t_{j}\right)+\left.\beta_{(k)}^{\prime \prime} u^{j}(x)\right|_{x=z \in \Gamma}}
\end{aligned}
$$

and then, by (22),

$$
\left.D_{\underline{v}}^{\beta_{(k)}^{\prime \prime}}(\underline{L u})^{i_{k}}\right|_{\Gamma}=g
$$

Case 2. Let $i \neq i_{k}, i^{*} \leq i \leq N$, and $\beta^{\prime \prime}=\left(0, \ldots, 0, \beta_{d+1}^{(k)}, \ldots, \beta_{n}^{(k)}+s_{i_{k}}-s_{i}\right)$. ( $\beta^{\prime \prime}$ is not a multi-index for $i_{r}^{*} \leq i<i^{*}$.) We get $\left|\beta^{\prime \prime}\right|=r_{0}-s_{i}+s-r$. We will show that

$$
\left.D_{\underline{v}}^{\beta^{\prime \prime}}(\underline{L u})^{i}\right|_{\Gamma}=0 \text {. }
$$

Now $\alpha+\beta^{\prime \prime}=\left(0, \ldots, 0, \beta_{d+1}^{(k)}, \ldots, \beta_{n-1}^{(k)}, \beta_{n}^{(k)}+s_{i_{k}}+t_{j}\right)$ only if $\alpha=\left(0, \ldots, 0, s_{i}+t_{j}\right)$. From (25) and (22), we obtain

$$
\left.D_{\underline{v}}^{\beta^{\prime \prime}}(\underline{L u})^{i}\right|_{\Gamma}=0 .
$$

Case 3. Let $i_{r}^{*} \leq i \leq N$ be arbitrary and $\beta^{\prime \prime}=\left(0, \ldots, 0, \beta_{d+1}, \ldots, \beta_{n}\right)$ be a multiindex with the following properties:

1. $\left|\beta^{\prime \prime}\right|=r_{0}-s_{i}+s-r$.

2. $\beta^{\prime \prime} \neq\left(0, \ldots, 0, \beta_{d+1}^{(k)}, \ldots, \beta_{n-1}^{(k)}, \beta_{n}^{(k)}+s_{i_{k}}-s_{i}\right)$.

(For $\beta_{n}^{(k)}+s_{i_{k}}-s_{i}<0$ this condition is always satisfied.)

3. There exists at least a pair $(j, \alpha)$ with $1 \leq j \leq N_{i}$ and $|\alpha|=s_{i}+t_{j}$ such that $\alpha+\beta^{\prime \prime}=\left(0, \ldots, 0, \beta_{d+1}^{(k)}, \ldots, \beta_{n-1}^{(k)}, \beta_{n}^{(k)}+s_{i_{k}}+t_{j}\right)$.

We will show that $\lambda_{i, \beta^{\prime \prime}}=0$. To this end, we show $\beta^{\prime \prime}<\beta_{(k)}^{\prime \prime}$ according to the order relation introduced in step 10. From (29), we get $\beta_{l} \leq \beta_{l}^{(k)}$ for $l=d+1, \ldots, n-1$. We claim that $\beta_{l}<\beta_{l}^{(k)}$ for at least one of these $l$ and prove this indirectly. So assume that $\beta_{l}=\beta_{l}^{(k)}$ for all $l=d+1, \ldots, n-1$. By (29), we get $\alpha_{l}=0$ for $l=d+1, \ldots, n-1$. Hence $\alpha=\left(0, \ldots, 0, s_{i}+t_{j}\right)$. Furthermore, $\alpha_{n}+\beta_{n}=s_{i}+t_{j}+\beta_{n}=\beta_{n}^{(k)}+s_{i_{k}}+t_{j}$; hence $\beta_{n}=\beta_{n}^{(k)}+s_{i_{k}}-s_{i}$, and thus

$$
\beta^{\prime \prime}=\left(0, \ldots, 0, \beta_{d+1}^{(k)}, \ldots, \beta_{n-1}^{(k)}, \beta_{n}^{(k)}+s_{i_{k}}-s_{i}\right),
$$

which contradicts (28). Therefore, $\beta_{l} \leq \beta_{l}^{(k)}$ for $l=d+1, \ldots, n-1$ and $\beta_{l}<\beta_{l}^{(k)}$ for at least one of these $l$. Hence $\beta^{\prime \prime}<\beta_{(k)}^{\prime \prime}$ and $\left(i, \beta^{\prime \prime}\right)<\left(i_{k}, \beta_{(k)}^{\prime \prime}\right)$. By the induction hypothesis (21), $\lambda_{i, \beta^{\prime \prime}}=0$. 
Case 4. Let $i_{r}^{*} \leq i \leq N$ be arbitrary and $\beta^{\prime \prime}=\left(0, \ldots, 0, \beta_{d+1}, \ldots, \beta_{n}\right)$ be a multiindex with the following properties:

1. $\left|\beta^{\prime \prime}\right|=r_{0}-s_{i}+s-r$.

2. $\beta^{\prime \prime} \neq\left(0, \ldots, 0, \beta_{d+1}^{(k)}, \ldots, \beta_{n-1}^{(k)}, \beta_{n}^{(k)}+s_{i_{k}}-s_{i}\right)$.

3. There does not exist a pair $(j, \alpha)$ with $1 \leq j \leq N_{i}$ and $|\alpha|=s_{i}+t_{j}$ such that $\alpha+\beta^{\prime \prime}=\left(0, \ldots, 0, \beta_{d+1}^{(k)}, \ldots, \beta_{n-1}^{(k)}, \beta_{n}^{(k)}+s_{i_{k}}+t_{j}\right)$.

From (24) and (25), we get $\left.D_{\underline{v}}^{\alpha+\beta^{\prime \prime}} u^{j}\right|_{\Gamma}=0$ for all $j$ with $1 \leq j \leq N_{i}$ and all $\alpha$ with $|\alpha| \leq s_{i}+t_{j}$. Hence, we get immediately

$$
\left.D_{\underline{v}}^{\beta^{\prime \prime}}(\underline{L u})^{i}\right|_{\Gamma}=0
$$

Thus (27) is proved. From (26), we get

$$
\left\langle g, \lambda_{i_{k}, \beta_{(k)}^{\prime \prime}}\right\rangle=0
$$

Since $\lambda_{i_{k}, \beta_{(k)}^{\prime \prime}}$ is an element of $\left(C^{r}(\bar{\Gamma})\right)^{\prime}$, and since $g \in C^{r}(\bar{\Gamma})$ is an arbitrary function, we obtain $\lambda_{i_{k}, \beta_{(k)}^{\prime \prime}}=0$.

Step 14. Hence, we get by induction

$$
\lambda_{i_{l}, \beta_{(l)}^{\prime \prime}}=0 \text { for all } l=1, \ldots, k_{1},
$$

and hence

$$
\lambda_{i, \beta^{\prime \prime}}=0 \quad \text { for } \quad i=i_{r}^{*}, \ldots, N \text { and }\left|\beta^{\prime \prime}\right|=r_{0}-s_{i}+s-r .
$$

Hence, from

$$
\left\langle(\underline{R u})^{\top}, \underline{T}\right\rangle=\sum_{l=r}^{r_{0}} \sum_{i=i_{l}^{*}\left|\beta^{\prime \prime}\right|=r_{0}-s_{i}+s-l}^{N}\left\langle\left. D_{\underline{v}}^{\beta^{\prime \prime}}(\underline{L u})^{i}\right|_{\Gamma}, \lambda_{i, \beta^{\prime \prime}}\right\rangle
$$

for all $\underline{u} \in C^{0, r_{0}+T+s}\left(\mathbf{R}^{n}\right)$, we can conclude that

$$
\left\langle(\underline{R u})^{\top}, \underline{T}\right\rangle=\sum_{l=r+1}^{r_{0}} \sum_{i=i_{l}^{*}}^{N} \sum_{\left|\beta^{\prime \prime}\right|=r_{0}-s_{i}+s-l}\left\langle\left. D_{\underline{v}}^{\beta^{\prime \prime}}(\underline{L u})^{i}\right|_{\Gamma}, \lambda_{i, \beta^{\prime \prime}}\right\rangle
$$

for $r<r_{0}$, and

$$
\left\langle(\underline{R u})^{\top}, \underline{T}\right\rangle=0
$$

for $r=r_{0}$ and all $\underline{u} \in C^{0, r_{0}+T+s}\left(\mathbf{R}^{n}\right)$. If we apply this conclusion first for $r=0$, and then step by step for $r=1, \ldots, r_{0}$, we obtain finally

$$
\left\langle(\underline{R u})^{\top}, \underline{T}\right\rangle=0
$$

for all $\underline{u} \in C^{0, r_{0}+T+s}\left(\mathbf{R}^{n}\right)$.

\section{Step 15.}

Let $\underline{f}=\left(\underline{f}^{1}, \ldots, \underline{f}^{N^{*}}\right)^{\top} \in \prod_{i=1}^{N^{*}} \prod_{\left|\beta^{\prime \prime}\right| \leq t_{i}+s-1} D_{i, \beta^{\prime \prime}}(\Gamma)$ be arbitrary. We will show that $\left\langle\underline{f}^{\top}, \underline{T}\right\rangle=0$. Because of property $(\mathrm{a} 2)$, there exists a sequence $\left(\underline{\phi}^{(l)}\right)_{l=1}^{\infty} \subset$ $\prod_{i=1}^{N^{*}} \prod_{\left|\beta^{\prime \prime}\right| \leq t_{i}+s-1} C^{\infty}(\bar{\Gamma})$ such that

$$
\left\|\underline{f}-\underline{\phi}^{(l)}\right\|_{\prod_{i=1}^{N^{*}} \prod_{\left|\beta^{\prime \prime}\right| \leq t_{i}+s-1} D_{i, \beta^{\prime \prime}}(\Gamma)} \longrightarrow 0 \quad \text { as } \quad l \rightarrow \infty .
$$


By applying Lemma 4 component-wise, we obtain for each $l \geq 1$, the existence of a vector function $\underline{u}^{(l)} \in C_{0}^{r_{0}+T+s}\left(\mathbf{R}^{n}\right)$ such that $\underline{R u}^{(l)}=\underline{\phi}^{(l)}$. From (30), we get

$$
\left\langle\left(\underline{\phi}^{(l)}\right)^{\top}, \underline{T}\right\rangle=\left\langle\left(\underline{R u}^{(l)}\right)^{\top}, \underline{T}\right\rangle=0
$$

for all $l$. Hence, since $\underline{T} \in \prod_{i=1}^{N^{*}} \prod_{\left|\beta^{\prime \prime}\right| \leq t_{i}+s-1}\left(D_{i, \beta^{\prime \prime}}(\Gamma)\right)^{\prime}$, we finally obtain the desired result

$$
0=\lim _{l \rightarrow \infty}\left\langle\left(\underline{\phi}^{(l)}\right)^{\top}, \underline{T}\right\rangle=\left\langle\underline{f}^{\top}, \underline{T}\right\rangle
$$

for all $\underline{f}$, hence $\underline{T}=\underline{0}$.

\section{References}

1. H. Beckert, Eine bemerkenswerte Eigenschaft der Lösungen des Dirichletschen Problems beilinearen elliptischen Differentialgleichungen, Math. Ann. 139 (1960), 255-264.

2. K. Beyer, Approximation durch Lösungen elliptischer Randwertprobleme, Rostock. Math. Kolloq. 26 (1984), 27-34.

3. — Zur Approximation durch Multipolpotentiale der Laméschen Gleichungen, Rostock. Math. Kolloq. 40 (1990), 29-34.

4. F. E. Browder, Functional analysis and partial differential equations II, Math. Ann. 145 (1962), 81-226.

5. $\ldots$, Approximation by solutions of partial differential equations, Amer. J. Math. 84 (1962), $134-160$.

6. U. Clauss, Approximation durch Normalableitungen von Fundamentallösungen elliptischer Differentialoperatorsysteme, Diplomarbeit, Universität Rostock 1994.

7. W. Freeden and R. Reuter, A constructive method for solving the displacement boundary value problem of elastostatics by use of global basis systems, Heidelberg Scientific Center TR 89.03.007, 1989.

8. A. Göpfert, Über $L_{2}$-Approximationssätze-eine Eigenschaft der Lösungen elliptischer Differentialgleichungen, Math. Nachr. 31 (1966), 1-24.

9. U. Hamann, Approximation durch Normalableitungen von Lösungen elliptischer Randwertprobleme in beliebigen Sobolev-Räumen, Math. Nachr. 128 (1986), 199-214.

10. _. Approximation durch Lösungen allgemeiner elliptischer Randwertprobleme bei Gleichungen beliebiger Ordnung, Habilitationsschrift, Universität Rostock 1986.

11. __ Approximation durch Lösungen elliptischer Randwertprobleme auf geschlossenen Hyperflächen, Math. Nachr. 136 (1988), 285-301.

12. __ Approximation mittels Linearkombinationen von Fundamentallösungen elliptischer Differentialoperatoren, Math. Nachr. 154 (1991), 265-284.

13. __ Approximation by linear combinations of fundamental solutions of elliptic systems of partial differential operators, Z. Anal. Anw. 13 (1994), 49-71.

14. V. D. Kupradze, Three-Dimensional Problems in the Theory of Elasticity and Thermoelasticity (in Russian), Moscow, Nauka, 1976.

15. I. Roitberg and Ja. A. Roitberg, On approximation of the solution of elliptic boundary value problems by linear combinations of the fundamental solution (in Russian), Doklad. Nauk. Ukrain. 12 (1992), 15-20.

16. Ja. A. Roitberg and Z. G. Sheftel, On the density of the solutions of elliptic boundary value problems in the sense of Petroskij systems in functional spaces on manifolds, in Symposium on Analysis on Manifolds with Singularities, Breitenbrunn, 1990, Teubner-Texte zur Mathematik, vol. 131, (eds. B.-W. Schulze and H. Triebel), Stuttgart-Leipzig, 1992, pp.176-180. 
17. B.-W. Schulze and G. Wildenhain, Methoden der Potentialtheorie für elliptische Differentialgleichungen beliebiger Ordnung, Berlin, Akademie-Verlag, 1977 and Basel-Stuttgart, BirkhäuserVerlag, 1977.

18. G. Wildenhain, Approximation in Sobolev-Räumen durch Lösungen allgemeiner elliptischer Randwertprobleme bei Gleichungen beliebiger Ordnung, Rostock. Math. Kolloq. 22 (1983), 43-56.

19. J. Wloka, Funktionalanalysis und Anwendungen, Berlin-New York, Walter deGruyter, 1971.

Department of Mathematics, University of Pennsylvania, Philadelphia, PA 19104-6395, U.S.A.

E-mail: uc@informatik.tu-cottbus.de

Fachbereich Mathematik, Universität Rostock, D-18051 Rostock, Germany

E-mail: uwe.hamann@mathematik.uni-rostock.de 